\title{
Robot Vision in Industrial Assembly and Quality Control Processes
}

\author{
Niko Herakovic \\ University of Ljubljana, \\ Slovenia
}

\section{Introduction}

In modern industrial assembly and quality control processes, that provide one of the crucial factors for the competitiveness of industry in general, there is a strong need for advanced robot-based object detection and recognition, object grasping and for the capability to perform assembling operations in non-structured environments with randomly positioned objects. Vision-based robotic assembly and quality control systems, that have been a topic of continued research interest for almost four decades, have now matured to a point where they can be effectively applied to advanced robot-based assembly and quality control tasks. This chapter will give an overview of research work related to the field of automated vision systems for assembly and quality control processes.

The nowadays' economy is more concentrated on producing customized products, however, and much less focused on mass manufacturing. In such an economy the need is far more dependent on ease of use, higher degrees of adaptation including assembling operations and control processes in non-structured environments with diversified and randomly positioned objects that enable small runs of made-to-order products. Automating a manual visual inspection process through a robot vision environment can create faster cycle times and level rates of throughput. Also robots will be ubiquitous in the industrial assembly of the future (table 1) and will support mass customization in smaller factories, where production runs are short, lot sizes small and products are modular in configuration and highly variable in features (Kellett, 2009).

\begin{tabular}{|c|c|c|}
\hline Robots in Manufacturing & Today & Future \\
\hline Production mode & Mass production & Mass Customization \\
\hline Production runs & Long & Short \\
\hline Lot sizes & Large & Small \\
\hline Product configurations & Non-modular & Modular \\
\hline Product features & Limited variety & Highly variable \\
\hline
\end{tabular}

Table 1. Robots in Manufacturing: Today Versus the Future (Kellett, 2009) 
As advanced robotic systems are becoming more popular and widespread in many industrial assembly settings, the need for reliable operation with the least possible amount of downtime is a common, expected demand. Traditional assembly robots are programmed to pick up a part from the exact same location every time and if the part is even slightly out of the place, the robot will fail to pick that part. Significant advantages can be realized when these robots are coupled with vision systems. Modern robot vision configurations with advanced recognition systems are used more often in later time to adjust the coordinates from where the robot expected to find the object to where it actually is located. This can be achieved with using only a single camera, multiple cameras or different combination systems. Cameras, computer and software work together with the robot to adjust the robot's position, allowing retrieval of the part. One of the examples in this direction is the continued improvements to $3 \mathrm{D}$ robot vision. The advances in 3D vision have made robots adept at recognizing a changing environment and adapting to it. This flexibility has allowed robots to work on projects that lack precise consistency, something that was very difficult for a robot to do in the past. Nowadays, robotic vision research is expanding into many new areas. Robots can now pick variously shaped objects from an indexing conveyor, eliminating the need for part designated in-feed systems and machines (figure 1). Research in this area has even made it feasible to pick from a box for certain part configurations (Christe, 2009).

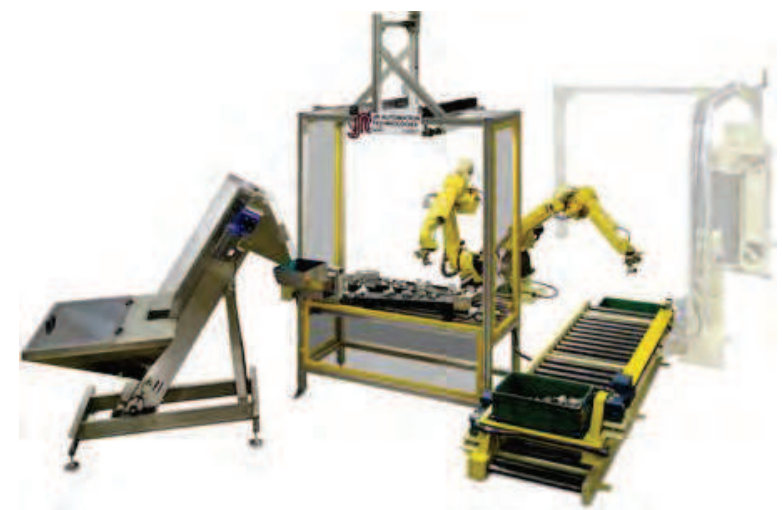

Fig. 1. Vision Guided Robotic Flexible Feeding (Christe, 2009)

Many current vision systems require extensive support from trained experts and are less reliable due to their complexity. This is the main reason why a fundamental step change to simplify the programming and mechanical complexity of robotic guidance applications is necessary. An innovative vision scheme is only half the battle. Mechanical reliability also plays an important role.

Implementation of robot vision in assembly and quality control processes is a multilayer problem and demands therefore expert knowledge, experiences, innovations and most often a problem specific solution. Usually, the procedure of the planning and development of a process of an assembly, inspection and measurement equipment using machine vision is split into precise determination of tasks and goals like detection, recognition, grasping, handling, measurement, fault detection, etc. and into machine vision component selection and working conditions determination like camera, computer, lenses and optics, 
illumination, position determination, etc. With regard to the automatic assembly part handling, robotic handling and assembly systems offer good prospects for the rationalization and flexibilisation of assembly and quality control processes.

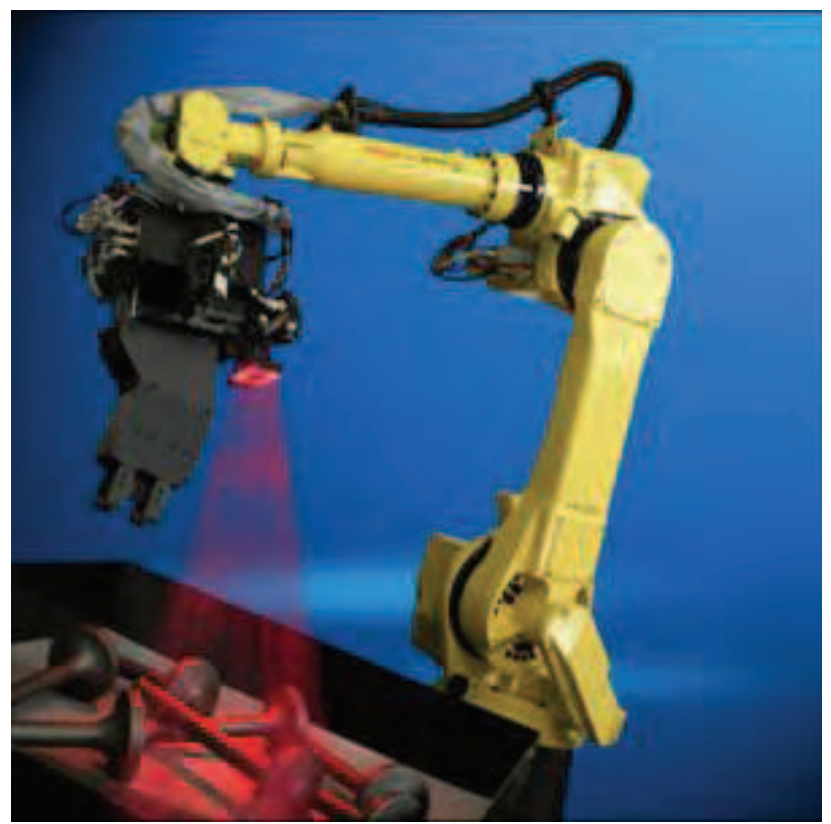

Fig. 2. Vision equipped robot in a bin picking application (Brumson, 2009)

Machine vision will certainly help take robot based assembly to the next level, and machine vision will probably be a part of the next generation safety solution. Off-the-shelf products already offer work cell protection based on 360-degree vision technology, and more robot controllers now come with built-in machine vision capability. Very frequently used operation in industrial assembly, a random part bin picking (figure 2), will probably benefit from such advancements, as will other complex tasks too (Brian, 2008).

\section{Industrial assembly}

Industrial assembly is a part of the production process (figure 3) and can be defined as an ordered sequence of physical handling tasks in which discrete parts and components are brought together and joined or mated to form a specified configuration. Assembly is a production process operation that provides a crucial factor for the competitiveness of industry in general. It is surprising, that such an important manufacturing process, that can take up to $30 \%$ of the manufacturing cost of an end product (Rowland \& Lee, 1995), is still mainly performed by hand. Manual assembly becomes expensive, if high levels of quality are to be achieved, because it involves highly skilled human laborers. Also much verification and inspection is needed to compensate for potential human insufficiencies. Manual assembly is often difficult, tedious and time consuming. 


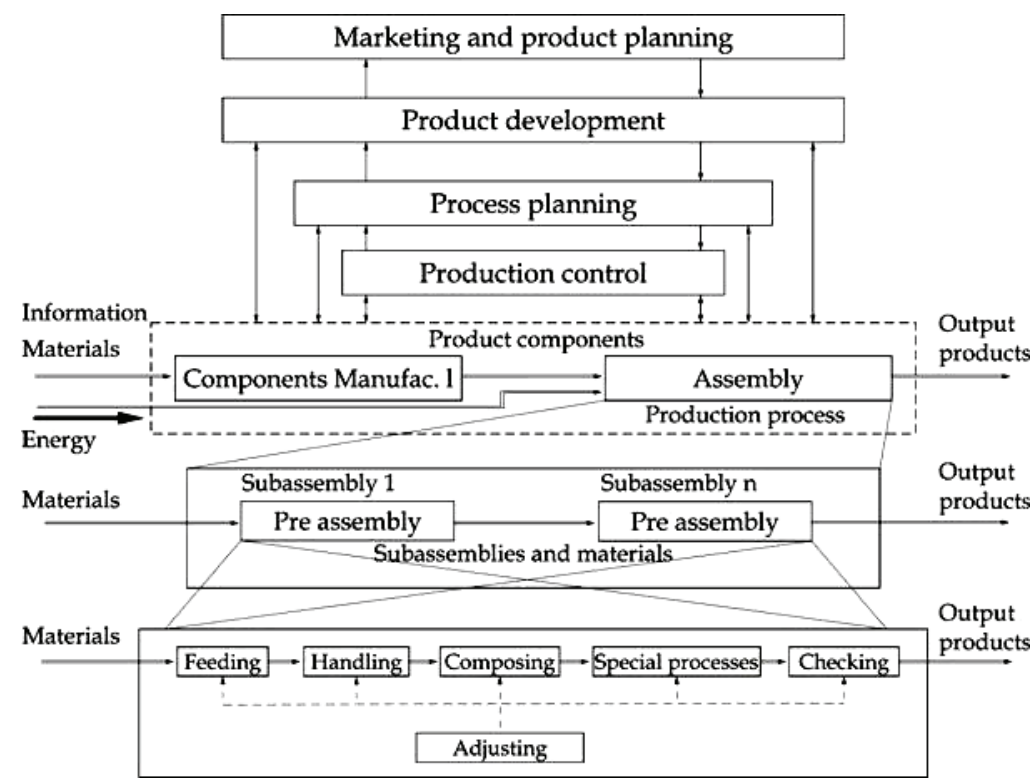

Fig. 3. Assembly as part of the production process (Rampersad, 1994)

For this reason it is often difficult for companies to follow market demands, when their assembly is mainly based on manual assembly processes. This becomes even truer, as the market requires products which satisfy high expectations in the areas of quality, price and delivery time. The key word in achieving this goal is the continuous refinement of product and production. Assembly is often the weakest point in the whole production process, because this activity takes up a substantial part of the total production costs and the throughput time (figure 4). The main reasons for this fact are the increasing labour costs, product variety and the decreasing product quantity.

Taking into consideration all these facts, together with constant price cuts for robots and overall turnkey systems in assembly and especially with continually improving performance of robots and machine vision systems, it is possible to understand the reasons for considerable grow of the area of robot-based assembly in the world in the last few years Robotic assembly offers good perspectives also in small and medium sized batch production (Handelsman, 2006; Kellett, 2009; Christe, 2009; Rowland \& Lee, 1995; Rampersad, 1994).

Assembly, as a part of production systems, involves handling of parts and subassemblies, which have mostly been manufactured at different times and possibly even in separate locations (Nof et al., 1997). Assembly tasks thus result from the requirement to join certain individual parts, subassemblies and substances such as lubricants and adhesives into final assemblies of higher complexity in a given quantity and within a given time period. 
a)

TOTAL TIME IN PRODUCTION

\begin{tabular}{|c|c|}
\hline $\begin{array}{c}\text { Other production } \\
47 \%\end{array}$ & $\begin{array}{c}\text { Assembly operations } \\
53 \%\end{array}$ \\
\hline & \\
\hline Mating, joining & $\begin{array}{c}\text { All other - feeding, } \\
\text { handling, supervision, } \\
\text { adjus.,inspect. } \\
50 \%\end{array}$ \\
\hline
\end{tabular}

TOTAL UNIT PRODUCTION COST

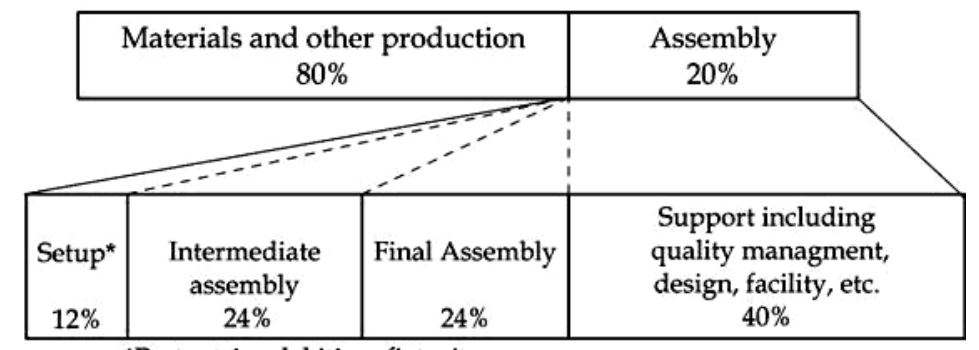

b)

Fig. 4. Typical average breakdown of a) production time and b) production costs of industrial products (Nof et al. 1997)

A typical assembly cell, which exact configuration may vary, will comprise of computercontrolled devices (robots, grippers, etc.), components and fixtures that can functionally accomplish or support one or all of the following tasks (Cecil et al., 2007):

- grasping of an organized/randomly positioned target part from a belt or a bin,

- manipulation and placement/assembly of parts,

- part and object recognition before, during and after assembly,

- quality control and inspection,

- planning and control of the actuators and grippers to accomplish the physical assembly.

Industrial robots nowadays can perform assembly and material handling jobs with very high speed and impressively high precision. However, compared to human operators, robots are hampered by their lack of sensory perception and their need for advanced sensorial capabilities in order to achieve more sophisticated tasks in a non-structured environment (King et al., 1988; Peňa-Cabera et al., 2005). In assembly processes, computer vision is often required to provide data to the applied robot systems in order to allow reliable grasping of objects and performing assembly tasks. Using a vision system for assembly often involves several challenges especially in the areas of data aquisition, coordinate transformation, invariant object recognition with vision systems as well as for the configuration and integration of vision systems into the robot environment. 


\section{Computer and robot vision}

Robot vision is concerned with the sensing of vision data and its interpretation by a computer and thus serves as a versatile robotic sensor. There is a growing demand requiring more complex and faster image processing capabilities in order to allow the implementation of vision systems into sophisticated industrial applications, like advanced assembly automation is.

Robot or machine vision is the application of computer vision to industry and manufacturing, mainly in robots. As computer vision is mainly focused on machine-based image processing, robot vision most often requires also digital input/output devices and computer networks to control other manufacturing equipment such as robotic arms (Davies, 2005). Specific advantages of robot vision systems include precision, consistency, cost effectiveness and flexibility.

Because of its primary mission, a computer vision is a part of a research discipline called artificial intelligence. Many methods, like neural networks and machine learning, developed in the field of artificial intelligence are used in computer vision. Computer vision is also linked with other research disciplines like neurophysiology, psychophysics, physics, computer graphics, digital signal processing etc. (Solina, 2006).

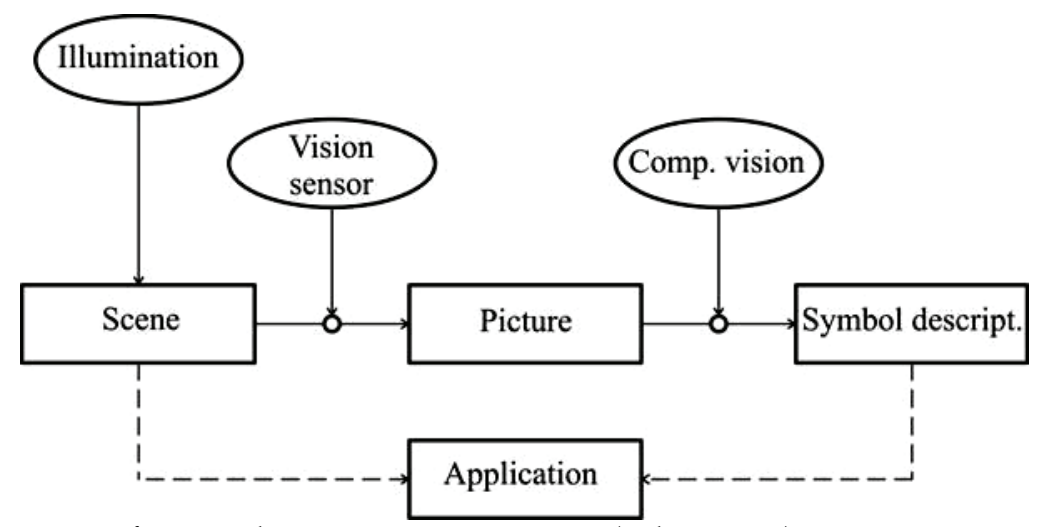

Fig. 5. Structure of a typical computer vision system (Solina, 2006)

Nevertheless, there are many research works being performed in this direction. In many cases in the area of assembly processes, especially in bin picking, a robot must perceive its 3$\mathrm{D}$ environment to be effective. Jet recovering 3-D information and describing it still remains the subject of fundamental research. Some research works (Gupta \& Knopf, 1993; Hou et al., 2007; Shapiro \& Stockman, 2001) are dealing with artificial vision systems based on the neural morphology of the biological human vision system, aiming to design computational neural structures and artificial vision systems following neural paradigms, mathematical models and computational architectures (Neuro -Vision Systems). Basic components of the computer vision system are presented in figure 5. Appearance of a 3-D scene depends mostly on illumination, position and the direction of the vision sensor. 
In a robot vision system, a variety of components are included. The systems layout mainly depends on factors like the environment, the application and the budget. Nevertheless, there are several common ingredients to all vision systems. A typical robot or machine vision system for assembly tasks consists of several of the following components (Davies, 2005; Griot, 2009; Batchelor, 2006; Batchelor \& Whelan, 2002):

- one or more digital or analogue cameras (black-and-white or colour) with suitable optics for acquiring images,

- a camera interface for digitizing images (frame grabber) - depends on the application,

- a processor (often a PC or embedded processor, such as a DSP) - when processors and frame grabbers are integrated into the camera itself, such cameras are called "smart cameras",

- input/Output hardware or communication links,

- optics - lenses to focus the desired field of view onto the image sensor,

- light source (LED, fluorescent or halogen lamps etc.),

- a program to process images and detect relevant features,

- a synchronizing sensor for part detection to trigger image acquisition and processing,

- actuators to sort or reject the processed parts.

Analog systems with classical frame grabbers have become well established in the marketplace and are built into many machines nowadays. The change from analog to digital technologies in the industrial image-processing sector causes that the classical frame grabber appears to be increasingly headed out to pasture. This is because digital communications interfaces such as Gigabit Ethernet, USB and FireWire allow for adequate camera-based solutions without image-capture cards (Leumann, 2009). The classical frame grabber is nowadays usually implemented in new projects primarily when a very short and calculable latency period plays a role in transmission of image data, such as for use with line scanning cameras or for implementation of high-speed cameras. They require data bandwidth from 25 to 70 megabytes per second, which exceeds the bit-serial standards such as USB 2.0, FireWire and Gigabit Ethernet. Additionally, the CameraLink Standard can achieve datatransfer rates up to $680 \mathrm{Mbps}$. So the classical frame grabber as plug-in card will have to support the CameraLink Standard for a long time to come.

Digital technology has some significant advantages in comparison to the analog one:

- digital image-processing systems allow significantly better image quality,

- up to 12-bit dynamic range,

- the camera's parameters can be set using software,

- the camera's availability, as well as its properties, can be maintained remotely,

- upgrading the camera in the field is easily achieved etc.

The most commonly used vision sensors in robot-based assembly are nowadays black-andwhite (or colour for some applications) CCD (charge-coupled device) cameras. 2D-vision systems consist of standard industrial CCD cameras used to take images that are processed by the robot to make decisions on how parts should be handled. Such systems are appropriate for parts, which are laying flat on a belt or in a bin with separator sheets. 
For parts that can stack upon each other or that may shift from side to side as the parts stack up or when parts are oriented randomly in a bin, depth estimation for a vision system is necessary. For depth estimation most commonly stereo vision systems are used. For such applications 3-D vision systems have to be applied to get a range image and the orientation of a part in 3D-space. Different laser sensors in conjunction with 2-D cameras, sensors with structured light and stereo cameras, together with different algorithms, can provide 3-D information (Baba et al., 2004; Thorsley et al., 2004; Schraft \& Ledermann, 2003). Even though these systems work well, stereo vision systems can provide inaccurate depth estimations, especially in cases with texture-less regions of images or in situations with insufficient illumination of the scene. Most of all, stereo vision is fundamentally limited by the baseline distance between the two cameras, which tends to provide inaccurate depth estimations, as the distances considered are getting large.

Different research approaches are addressing the depth estimation problem by using monocular visual cues, such as texture variations and gradients, defocus, colour etc. With applying a Markov Random Field learning algorithm to capture some of these monocular cues and with incorporating them into a stereo vision system, a significantly better accuracy in depth estimation is obtained than it is possible using either monocular or stereo cues alone (Saxena et al., 2007 a; Saxena et al., 2007 b).

Some other papers treat monocular 3-D vision and object pose estimation in a robot assembly environment. The approach described by L. P. Ray (Ray, 1990) is based on the estimation of the three dimensional position and orientation of objects from one or more monocular images on the prerequisite that the identities of the objects are known and that the three dimensional geometrical models are available. The main weakness of these solutions is the fact, which they fail to achieve the real time performance, necessary in many assembly applications. A solution of this problem is proposed by (Winkler et al., 1997). In this research approach a feature map strategy for the real time 3-D object pose estimation from single 2-D perspective views is presented. Based on the neural network and the systematic training of the Kohonen's self-organizing feature map, the method satisfies the accuracy requirements of object pose estimation in more than $90 \%$ of all considered cases.

With the intention to achieve an economic and flexible automatic assembly system working with a SCARA-robot, operating in a random environment, some research activities present the use of simple 2-D CCD cameras in conjunction with additional force sensors and embedded fuzzy sliding mode controllers (Scharstein \& Szelinski, 2002). The experimental results prove that the robotic motion control performance is good enough for executing the investigated assembly tasks.

The paper of Sharstein and Szelinski offers a very good overview of stereo vision systems and algorithms, developed by different researchers over the past few decades. In their work the authors present the taxonomy of dense, two-frame stereo methods, compare existing stereo methods and present experiments evaluating the performance of many different variants.

In robotic assembly, vision sensors have a different role than - for example - in mobile robots, where the tasks usually involve exploration of the environment. A robotic assembly 
cell represents a relatively well ordered environment and is part of an integrated manufacturing process, rather than operating in isolation. This facilitates the fulfillment of some of the major requirements for effective robot assembly. This is especially helpful for applications where expensive and complex machine vision systems should be avoided. The application of vision systems in robot-based assembly systems can be simplified, when products and components are designed for a robot-based assembly and if parts are fed to the system with a relatively accurate position and orientation (Boothroyd, 2005).

The slightly asymmetrical screwed part (figure 6) would not present significant problems in manual handling and insertion, whereas for automatic handling, an expensive vision system would be needed to recognize its orientation. In fact, it can be said that one of the possible benefits of introducing automation in the assembly of a product is, that it forces a reconsideration of its design, which might not only facilitate the part recognition but might also be used to implement other cost-saving or quality-related improvements (e.g. Poka-Yoke design).
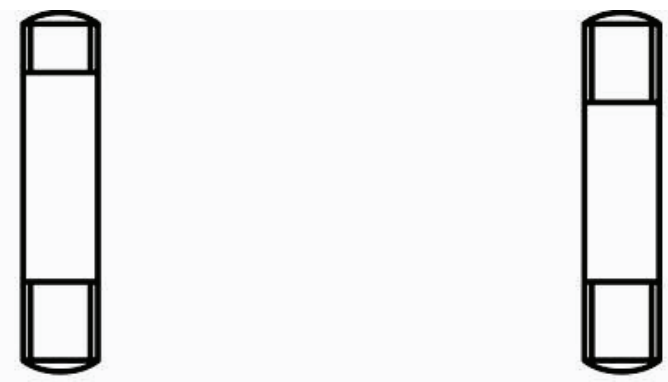

\section{Asymmetrical - difficult to orient $\quad$ Symmetrical - easy to orient}

Fig. 6. Design change to simplify automatic feeding and orientation (Boothroyd, 2005)

The major role of vision sensors in a robot assembly cell is to compare reality with expectations and to evaluate discrepancies. Essentially this means detecting presence or absence of correct parts and measuring to allow for the detection of component tolerances and positioning errors during relevant stages of assembly.

On one hand, many industrial assembly applications are successfully being handled nowadays using computer vision and robots, especially in robot-based assembly cells. However, for these applications objects have a simple (2D) shape and/or are organized in a structured manner. On the other hand, a general so-called »Bin-picking", where the objects have a 3-D shape and are randomly organized in a box, still remains a problem. Despite of many research works, that offer special solutions and improvements in the overcoming the bin-picking problem (Schraft \& Ledermann, 2003; Kirkegaard, 2005; Kirkegaard \& Moeslud, 2006; Hema et. al., 2007), the oldest challenge in robotics remains still unsolved.

\section{Object recognition}

In robot assembly, a vision recognition system aims to mimic the human sense of vision and must be capable of perceiving and detecting assembly parts as good as the humans can. Three-dimensional object recognition entails representation of a 3-D object, identification of 
the object from its image, estimation of its position and orientation and registration of multiple views of the object for automatic model construction. Important stages in the design and development of a recognition system are presented in figure 7 .

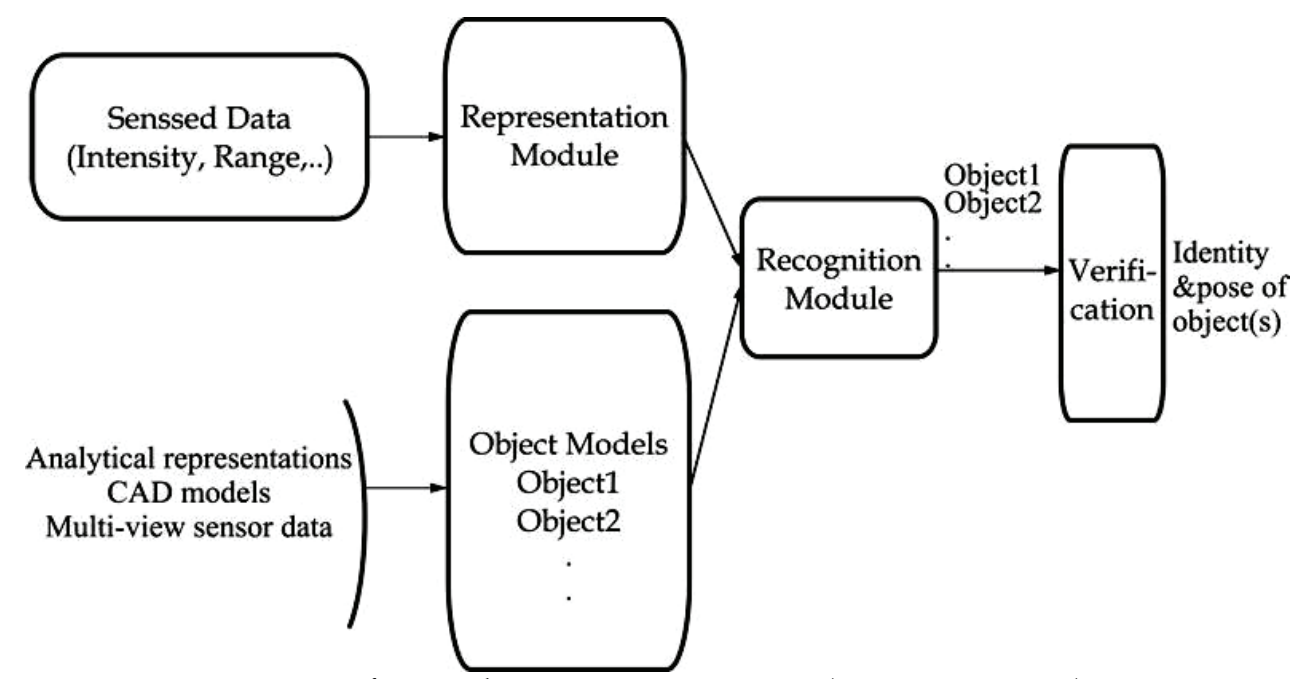

Fig. 7. Key components of a 3-D object recognition system (Jain \& Dora, 2000)

A typical approach for handling the object recognition tasks using traditional image processing and computer vision methods usually consists of five steps (Kirkegaard, 2005; Jain \& Dora, 2000; Pope, 1994; Faugeras, 1993; Yli-Yaasaki \& Ade, 1996):

- Detection or pre-processing - is the low level signal processing which extracts information from the scene and represents it as some form of primitive symbols (Motai \& Kosaka, 2004; Pope \& Lowe, 2000; Pope \& Lowe 1996; Roth et al., 2002; Vujovic et al., 2007).

- Grouping or segmentation - is based on the low level symbols, the primitive features are grouped into higher order features, which give more information for the selection and matching in the next steps (Lowe, 1987; Balslev \& Eriksen, 2002).

- Indexing or feature extraction - selecting from the stable object features the most likely model from a library of models (model base) and finding a way of quickly comparing a set of features with a model, avoiding a search through all models. As a critical component, a stable, representative feature extraction system should be developed to extract key features for a specific problem domain in the feature extraction stage. The result of feature extraction is normally a feature vector (Kirkegaard, 2005). There is usually one of two methods of representation applied:

- appearance-based approach - information about appearance of the object is used (Pope, 1994; Balslev \& Eriksen, 2002),

- model-based methods - information about geometrical features, type and spatial relations of the object is used (Motai \& Kosaka, 2004; Lowe, 1987).

- Matching or classification - finding the best fitting between the scene features and the model features and solving the localization problem. In this stage, a classifier system uses extracted key features to distinguish the classes of the objects of interest. The algorithms 
or methods for these stages are generally domain dependent, particularly when using traditional image processing and computer vision techniques. Learning paradigms such as neural networks or genetic programming approaches have usually been applied to the matching or classification stage Kirkegaard, 2005).

- Verification - verifying the estimated identity and location of an object (Kirkegaard, 2005, Pope \& Lowe, 1996).

To facilitate the task of identification and localization, a description of each object to be recognized is available to the computer and can be used. These descriptions can either be model-based or appearance-based, or a combination of both.

Based on the dimensionality of their spatial description, various types of object recognition problems can be stated (Antrade-Ceto \& Kak, 2000):

- recognition of a 2-D object from a single 2-D image,

- recognition of a 3-D object from a single 2-D image,

- recognition of a 3-D object from a single 3-D image (a range map),

- recognition of a 2-D or 3-D object from multiple 2-D images taken from different viewpoints, etc.

In the past decades, much progress has been made in the research of recognizing 2-D objects in single 2-D images and in recognizing 3-D objects in range maps. A considerable progress has also been made in the recognition of 2-D or 3-D objects using multiple 2-D images, as in binocular or stereo vision. However, today object recognition remains largely unsolved and is still a very active field of research.

The data of assembly parts is usually obtained by a CCD camera, giving intensity data or by laser line scanning which gives a range or depth map. From this data, features have to be extracted for object recognition, which involves the matching of image features from either range or intensity data, against a previous internal representation of the object (a model).

Certain features like curvatures are independent of the view point for the object. After inspection of respective signs of an object curvature using different approaches, it is possible to define the local surface area as being one of eight fundamental primitive types: peak, pit, ridge, valley, saddle ridge, saddle valley, minimal and flat. Different algorithms have been proposed to extract curvatures from an object (Fischler \& Bolles, 1986; Rosin \& West, 1995, Cox et al., 1993). The difficulty of these approaches is that extracted curvatures are highly dependent on the selected starting points and the order of the edge linking. Some other approaches (Alter \& Basri, 1998) propose dynamic programming and relaxation to avoid such problems. In this case the edges are organized as nodes of a graph and linked to each other through graphs.

Other common features, which are extracted from objects are edges, planar regions etc. Edge detection is a very important step in low level image processing and can be used in measurement of a distance from the obstacle. In (Vujovic et al., 2007) a new approach is proposed, which promises much faster edge detection capabilities as previously known edge detectors can provide. 
Many different approaches and methods have been developed or tackled in the field of object detection and recognition by different researchers in the past years. A quite detailed survey of previous work has been treated by (Jain \& Dora, 2000) and (Antrade-Ceto \& Kak, 2000). The authors discuss the recognition problems, methodologies and algorithms like object shape complexity, size of object model database, learning, individual and generic object categories, non-rigidity of objects, occlusion and viewpoint-dependency, object representations and recognition strategies (image sensors, models, matching strategies) and 3-D free form object recognition.

A very important field of research in object recognition is represented by the area of learning/adaptive algorithms. One of the major advantages of a learning system is the ability to learn/extract the useful features from the training data set and to apply these features to the test data. Some authors (Roth et al., 2002) use a PAC (Probably Approximately Correct) model for their learning strategies. In this research, authors quantify success relatively to the distribution of the observed objects, without making assumptions on the distribution. In (Pope \& Lowe, 1996) authors model the appearance of an object using multiple views, including a training stage, in which the system learns to extract the models characteristics from training images and recognizes objects with it. The model uses probability distributions to characterize the significance, position, intrinsic measurements of various discrete features of appearance and also describes topological relations among features. A matching procedure, combining qualities of both iterative alignment and graph matching uses feature uncertainty information recorded by the model to guide the search for a match between model and image.

In recent years, since the late 1980s, neural and genetic learning paradigms (neural networks, genetic paradigms and genetic programming) have attracted attention as very promising methods of solving automatic target recognition and detection problems. In particular, neural and genetic systems offer potentially powerful learning and adaptive abilities and are very suitable for automatic object recognition in real time (Winkler et al., 1997, Klobucar et al., 2007).

In general, the currently available computer vision systems for object recognition are still not as adaptive and universal as biological systems are. Successful commercial systems of computer vision are usually designed to solve well defined and/or specific tasks. For solving tasks computer vision systems often combine multiple standard strategies or even apply strategies that have been developed individually for a specific application (Forsyth \& Ponce, 2002).

In robot-based assembly production processes, there is very often a need for grasping and manipulating of complex objects. Most of the robot vision systems require the complete knowledge of both, the shape and the position of the assembly parts. Due to the fact that vision systems are often specifically adapted to the individual 2D-shapes and the individual way the parts are fed to the system, a change of the produced parts usually requires a time consuming adaptation of the overall system. This could represent a cost problem especially for small series. With respect to the before mentioned aspects, there is a need for efficient robots with advanced machine vision capabilities that allow the recognition of complex, and 
randomly organized parts, lying on a conveyor belt or in a bin, with little or no prior knowledge about the pose and geometry of the parts. Especially the so called bin-picking problem - the picking of randomly organized 3-D parts in a bin - has not yet been solved in a general manner, primarily due to severe occlusion problems.

Many researchers are dealing with this basic problem, which represents one of the remaining obstacles to a widespread introduction of vision systems to robot-based assembly. In searching for appropriate solutions, the above mentioned methodologies and algorithms for object detection and recognition are applied. In (Boughorbel et al., 2003; Goldfeder et al., 2007) range maps have been applied for the reconstruction of objects using super quadric representations and decomposition trees. Based on laser scanners for 3-D object detection a model based approach in combination with CAD models (Schraft \& Ledermann, 2003; Kristensen et al., 2001) or Harmonic Shape Context features (Kirkegaard, 2005; Kirkegaard \& Moeslund, 2006), which are invariant to translation, scale and 3-D rotation, have been applied. Also some research has been done applying stereo vision together with a set of two neural networks (which are then compared with each other) namely the Radial Basis Function nets and Simple Feed forward nets (Hema et al., 2007). An algorithm for segmentation of partially occluded bin objects and the location of the topmost object is proposed. The experimental results have shown that the proposed algorithm might be appropriate for many bin picking applications if special attention is paid to the lighting situation. The results of the above mentioned research in bin-picking are very promising but the research is still more or less in research stage. Even though that grasping is not optimal and for some object not feasible, all contributions show an improved accuracy and efficiency in bin picking and sorting.

\section{Applications of robot vision in control processes}

Introduction of robot vision control to an assembly process initially demands a clarification of the purpose of the application, which is usually not just a problem of measuring one or more product parameters in a very short time, but also fault detection, usually detected in manual assembly by operators, based on an appropriate sample.

In many assembly processes, the control process is still undertaken by the operators. They are capable of making an estimation and judgment about the accuracy and shape faults of a product or part using their acquired skills for quick and accurate decisions based on the human vision system. These tasks are usually complex and the accuracy and speed depend on the operator's psychological and physical condition. The consequences are unreliable results and a very serious possibility of overlooking faults. The reliability of human decisions is also reduced by the monotony of the task and by tiredness (Herakovic, 2007a).

Today's robot vision and identification systems are more and more reliable and robust, and therefore convenient for industrial applications and are becoming indispensable to the assembly or disassembly process (Braggins, 2006; Ostojic et al., 2008). Control systems, based on robot vision, are capable of maintaining a control process even more efficiently than a human if the conditions for an appropriate use of the technical benefits of robot vision are ensured (West, 2006, West, 2009). 
A robot vision system, which is used in an industrial environment for tasks such as inspection, measurement and fault detection, has to be a robust and very reliable system. For this reason, the development of measurement equipment using robot vision has to follow a fixed procedure (Trdic, 2000; Skvarc, 2000). Usually, the procedure is split into a precise determination of tasks (measuring, fault detection) and goals, into the robot vision selection and working conditions (illumination and position determination), component selection of the robot vision (camera, computer, lenses and optics), and finally, the development of an automatic robot handling system for the parts.

\subsection{Fault detection and dimension control}

When using a robot vision system for fault detection and dimension control, lighting is always a critical component and should be treated as one of the first steps of component selection. The effectiveness of an illuminating source in an inspection and fault detection process is determined by the direction at which light strikes an object and the direction of the reflected light into, or away from the camera. Suitable illumination covers the required field of view, creates a consistently measurable degree of contrast and does not cause reflected glare. Different light types are required to provide successful illumination for the broad range of products being inspected by automated systems (Merva, 2009).

Let us take an example of a robot vision application in quality control processes during the electromotor (EM) stator assembly. To get the optimal conditions for the effective robot vision quality control, some experimental research efforts of the robot vision tasks determination and especially of the influence of lighting on the fault detection and dimension control of electromotor (EM) stator are considered (Herakovic, $2007 \mathrm{~b}$ ).

In control process, final product quality control is in fact an inspection of parts and of final product in technical and visual manner. The challenge is greater when the product is composed of parts, produced by an unstable process. The EM stator, assembled from thin metal plates, represents such an example (figure 8). The assembling process is performed by a well known special forming machine. High accuracy and no visible faults on the EM stator are demanded so the 100 percent control process should be guaranteed by the robot vision. Assembly process is followed by painting and drying, and in both processes inaccuracy and fault can occur. The control process involves:

- EM stator height measuring,

- slot width measuring,

- spherity or so called had-shape control

- position and rotation of claw,

- painting quality - uniformity,

- slot occurrence on the roll outside,

- plate derivation and

- randomly, the inside and outside diameters of EM stator have to be measured. 


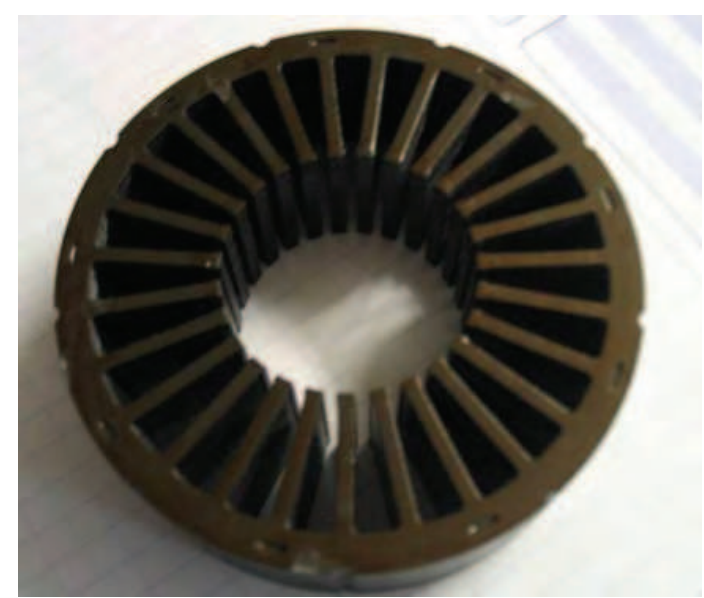

Fig. 8. EM stator assembled from thin metal plates

Mechanical defects on the inside and outside of EM stator, as a result of non-adequate assembly process, are shown in the figures $9 \mathrm{a}$ and b. Such defects are slot occurrences between plate (inside or outside) and broken EM stator.

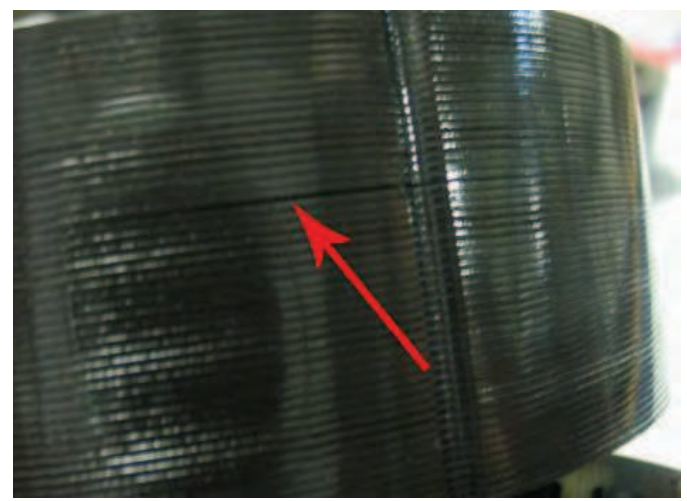

a)

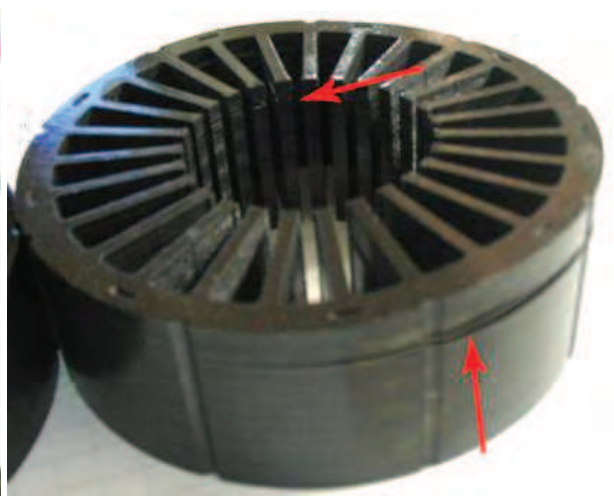

b)

Fig. 9. Slots inside and outside $(a, b)$ and broken EM stator (b)

Figure 10a shows mechanical damages, such as ruptured and derivate plats (can occur in performing the manual control by mechanical caliber). The derivations of plates inside of the EM stator are usually effected by assembly process (figure $10 \mathrm{~b}$ ). The appearance of varnish or paint on the EM stator also means a false product (figure $10 \mathrm{~b}$ ). 


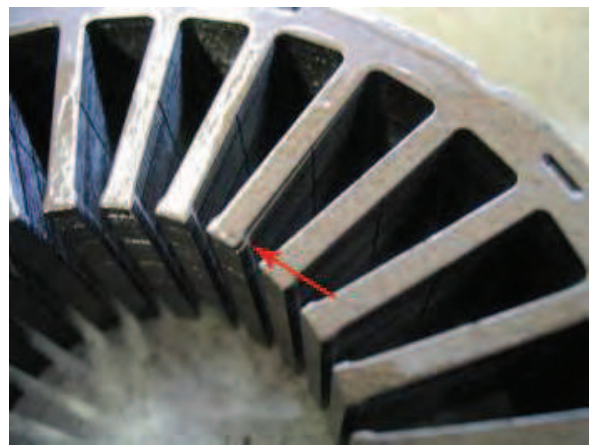

a)

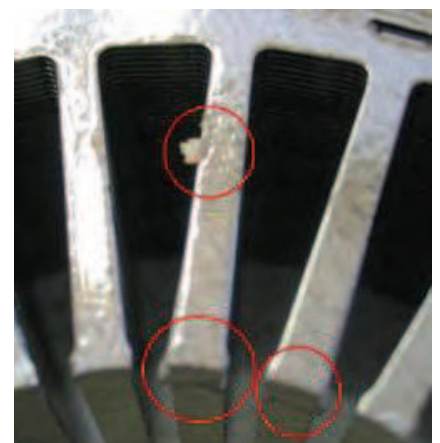

b)

Fig. 10. Moved and derivate plates $(a, b)$ and paint rest $(b)$

Manual control process of the above described critical places of the EM stator has been recognized as not reliable enough and is even a source of new damages of the finished product. The control process is also a bottleneck in the assembly process. In such a case the automation of the inspection and measuring of the EM stator can be the optimal solution for a reliable quality assurance.

The analysis of necessary conditions for the robot vision application in final quality control of the EM stator package can give very satisfying results through the experimental approach related to the technique and the type of illumination, which can guarantee a reliable robot vision quality control. For each critical area of the EM stator, the conditions which can influence the successful machine vision fault detection should be examined in grater detail. In the presented research this has been done by using the variation of influential parameters like the position of the camera and light source (Burns, 1999), type of the camera lenses (Jenny, 2000) and above all by the variation of the angle of the stator position regarding to the camera and the light source position. In the research the Sony XC-ST50-CE camera has been used.

Each critical area of the controlled stator demands a specific experimental setup, which must allow the analysis of all variations of different parameter influencees on the quality of the robot vision control of the stator. The aim of all presented experimental setups is to assure satisfactory quality of the detection of as many faults as possible, with a minimum number of handling operations of the stator and with the least possible changes of the type and sort of lighting and camera lenses.

Figure 11a represents an experimental setup for the analysis of the impact of a direct general-purpose different colour illumination (low angle ring illuminator) and the impact of different colour backgrounds on a depth control (for the reason of the light reflection), contrast and on a detection of visual defects of the EM stator. It is supposed that different background and illumination colours are more suitable for the visibility of some surface defects like remains of varnish and mechanical damages. 


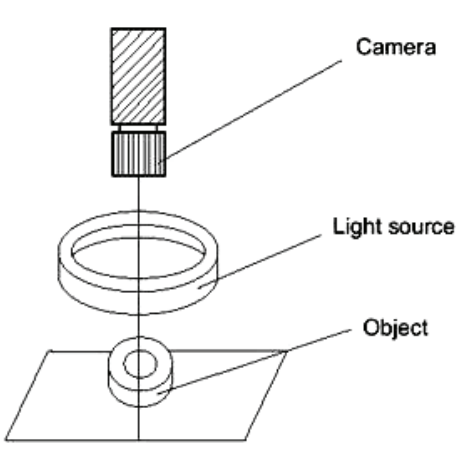

a)

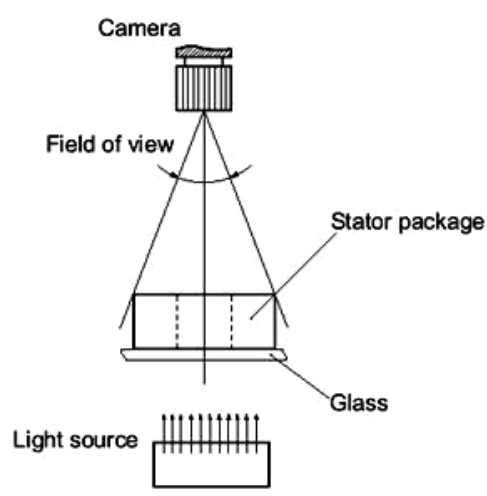

b)

Fig. 11. Experimental setup: a) general-purpose different colour illumination and different colour background; b) backlight illumination

Experimental setup with the backlight illumination (figure 11b) enables the analysis of parameters like the rapture of the stator plates, dimensions of the stator slots, general stator dimensions etc. The presented experimental setup is suitable for the reliable detection of the many of before mentioned parameters only with the use of appropriate camera lenses (conventional, telecentric etc.). In this case the type of lighting is not so important for the quality of detection.

For the detection of some parameters like mechanical damages on the outer stator circumference and slot occurrence as well as plate derivation and shifting on the inner stator circumference, the experimental setups presented in figure 12 are convenient. Also in this case the reliability of the detection of before mentioned parameters depends on the use of appropriate camera lenses; however, the type of lighting is decisive for the quality of detection this time.

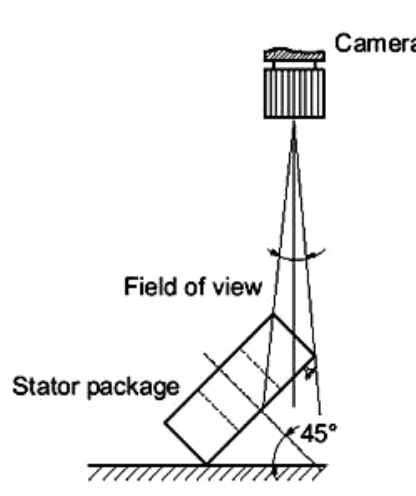

a)

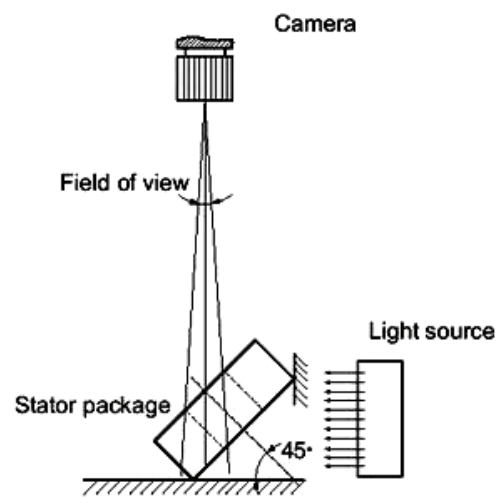

b)

Fig. 12. Fault detection on a) outer stator circumference and b) inner stator circumference 
As it is evident from the figure 12, one of the key parameters for the satisfactory quality detection of the stator faults is beside the camera lenses and lighting, also the angle of the stator inclination and the distance of the camera from the observed field. It is necessary to reach the best possible sharpness of the stator on the screen. The incline of the stator demands also the best possible depth sharpness, which is theoretically better when the incline of the object is bigger.

Using the experimental setup in figure 13a, an exact determination of spherity, height/width, parallelism and perpendicularity of the stator package is possible. The quality of the detection depends mainly on the type of lenses used and a little bit less on the type and the colour of the illumination.

In the case of the EM stator the inclination is limited with its geometry. The maximal theoretic inclination angle of the stator is $60^{\circ}$ as shown in figure $13 \mathrm{~b}$. If the inclination angle is bigger, the upper edge of the stator covers over the lower edge of the inner circumference and the camera filed of view can not cover the whole lower half of the inner circumference of the stator. For this reason more detailed experimental analysis of the influence of the stator inclination angle on the quality of the fault detection must be carried out with the consideration of the optimal distance of the camera from the observed object to get the largest possible filed of view at a time.

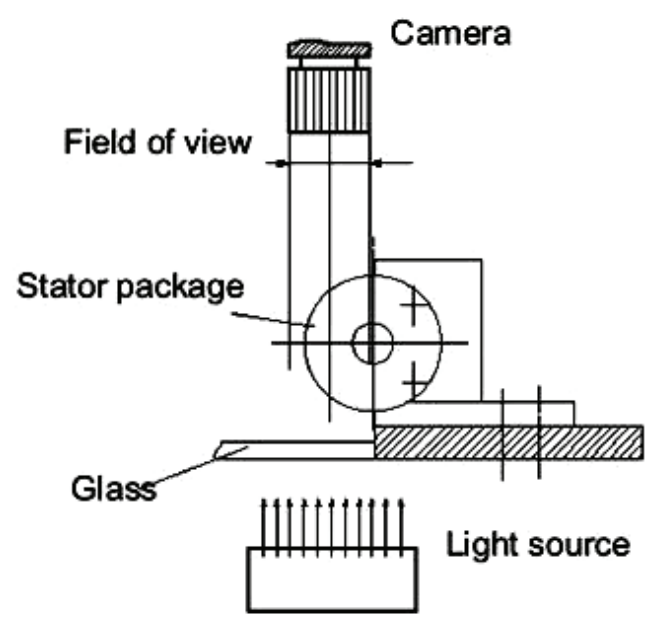

a)

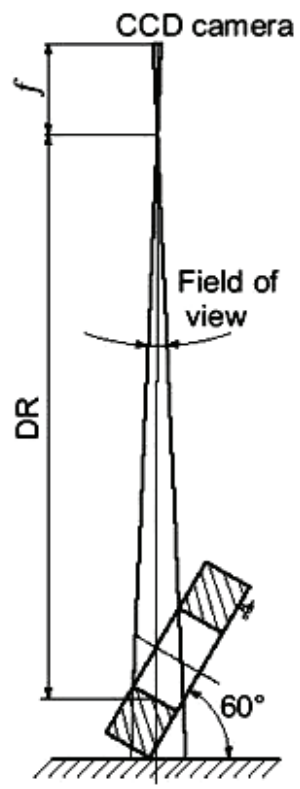

b)

Fig. 13. a) Spherity and dimension control; b) Maximal stator inclination angle 


\subsection{Experimental results}

With the results obtained from the above presented experimental research work it is possible to identify necessary conditions regarding the technique and the type of illumination as well as the inclination angle of the stator package to get the desired results with the robot vision quality control of the dimensions, mechanical damages and visual look of the EM stator.

Figure 14 shows the influence of the stator inclination angle on the fault detection quality. The increase of the inclination angle brings on better detection quality mainly of both edges of the inner stator circumference.

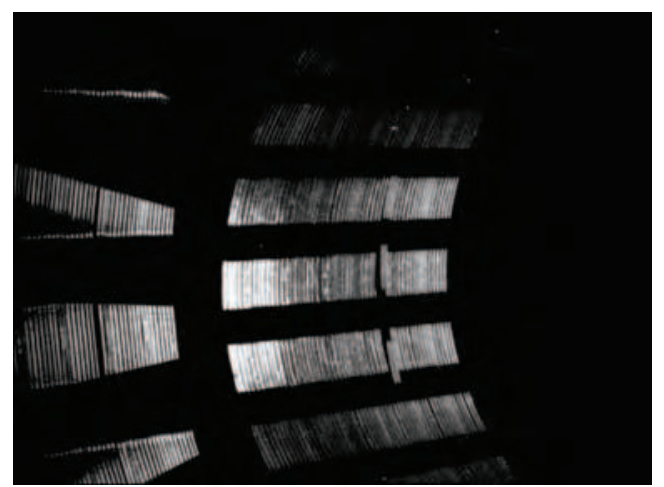

a)

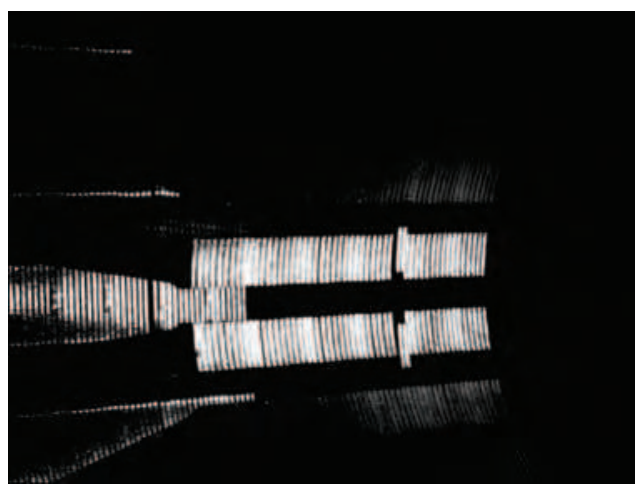

b)

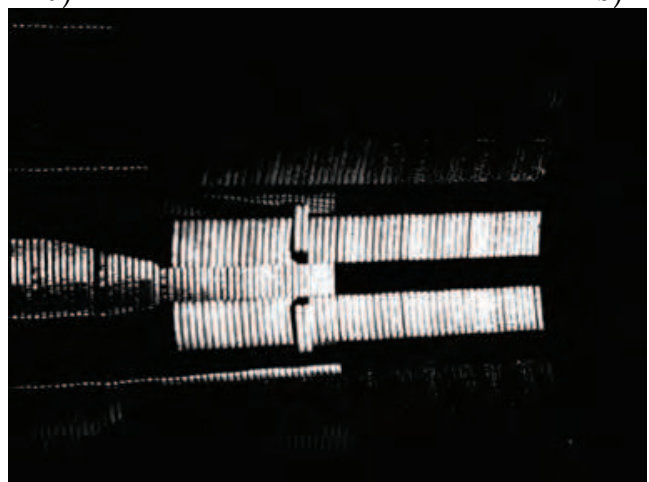

c)

Fig. 14. Impact of the stator inclination angle a) $30^{\circ}$, b) $45^{\circ}$ in c) $55^{\circ}$

However, the increase of the inclination angle causes the difference in the distance between the lighting source on one side and the upper and lower edge respectively of the observed segment on the other side. It has for consequence a non homogeneous illumination of the stator and herewith unreliable fault detection, as shown in figure 15. 


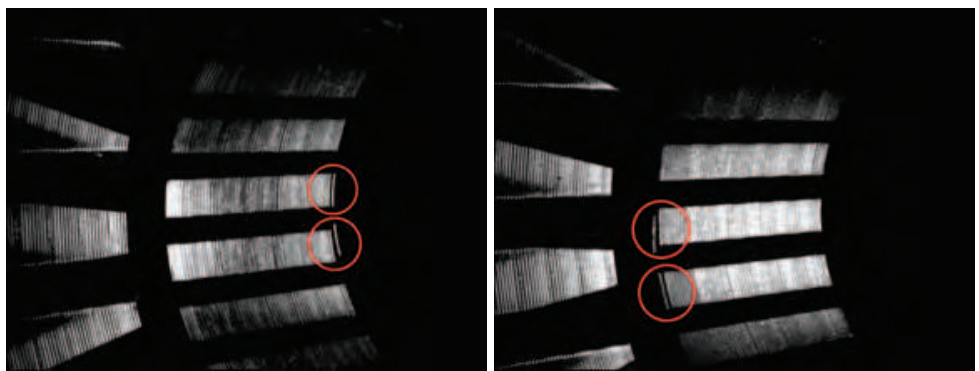

a)

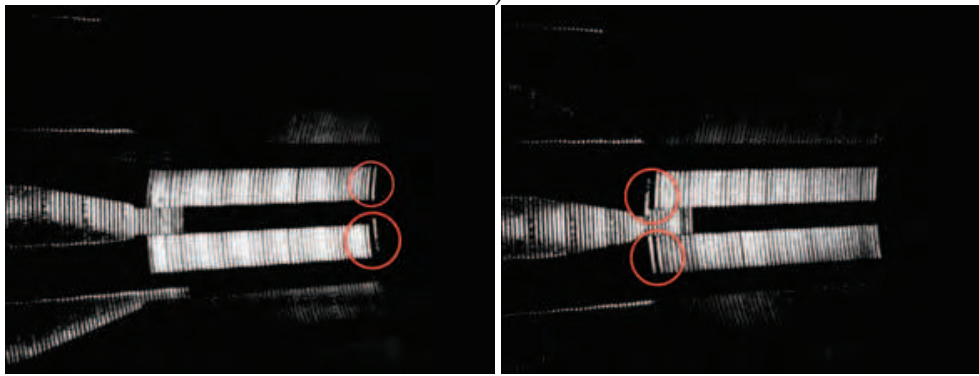

b)

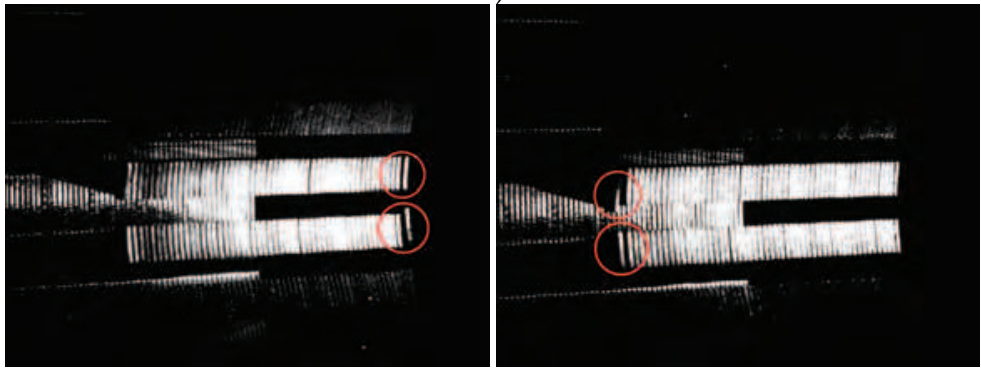

c)

Fig. 15. Influence of non homogeneous illumination: a) $30^{\circ}$, b) $45^{\circ}$ in c) $55^{\circ}$

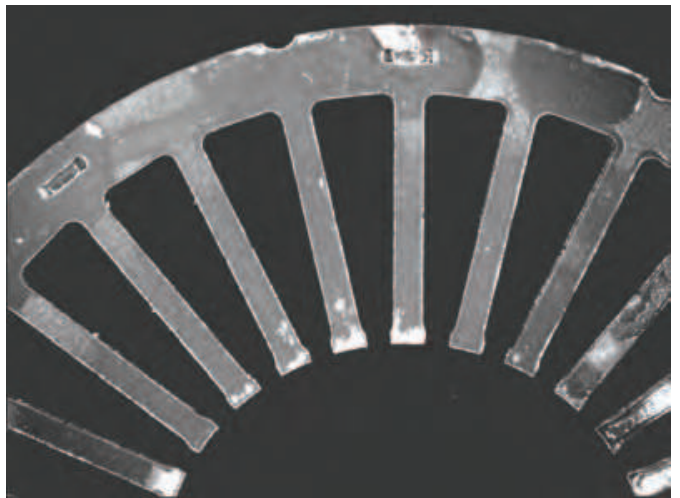

Fig. 16. Front surface defects - varnish accumulation 
One of the experimental results, using a low angle ring illuminator (figure 11a) with different colour lighting and different background colours, is presented in figure 16. In this case a black background colour was applied. Bright areas of the stator represent surface defects as a consequence of varnish accumulation or nonuniformity of varnish on the surface. The method is appropriate for the control of the front surfaces regarding the detection of the varnish nonuniformity.

The best results of the control of one of the most important dimension parameters of the stator, of the slot width, are possible with the back light illumination method (figure 11b) and with the use of a telecentric lenses. Figure 17 shows one of the experimental results, where it is obvious that the use of a telecentric lens (figure 17a) eliminates the negative effect of the depth, a parallax, which appears with the conventional lens (figure 17b).

The stator appears on the screen as 2D picture in ground plan with clear and sharp edges. This fact assures the $100 \%$ control of the slot width along the whole depth of the stator package. With this method also the $100 \%$ control of the inner and outer diameter of the stator package is possible.

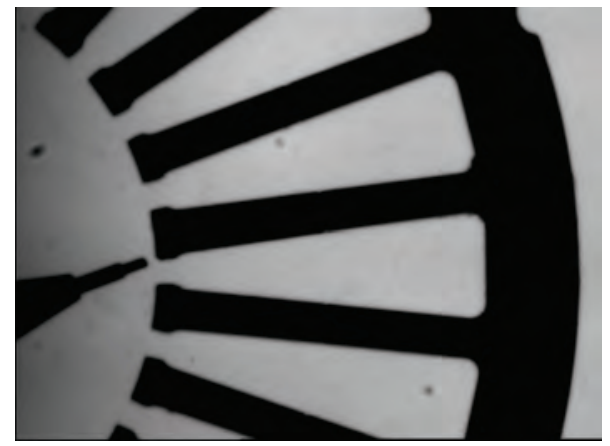

a)

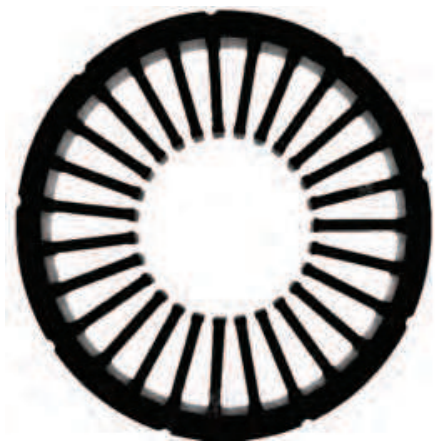

b)

Fig. 17. Control of the slot width with: a) telecentric and b) conventional lens

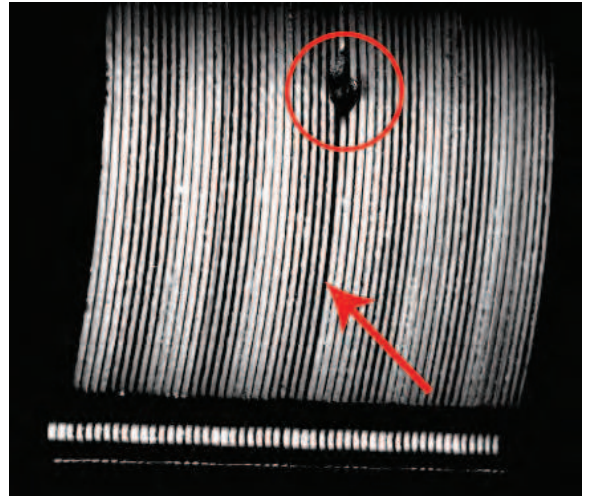

a)

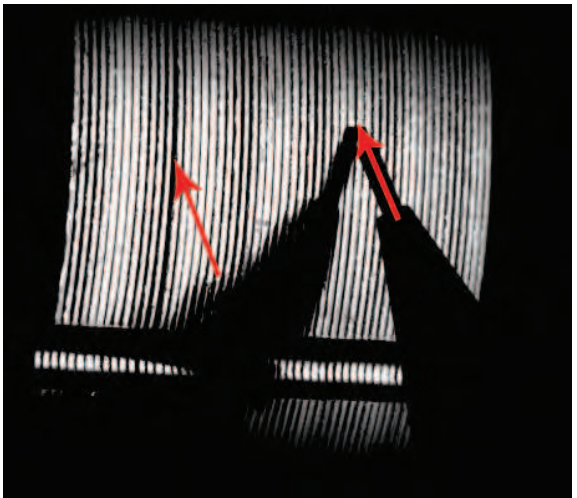

b)

Fig. 18. Mechanical damages (a) and slots (b) on the outer stator circumference 
Results of the experimental detection of the mechanical damages of outer and inner stator circumference with the experimental setup from the figure 12 are presented in figures 18 and 19, where a mechanical damage (figure 18a) and slots between the stator plates on outer circumference (figure 18b) are very well visible. Slots and plate derivations on the inner stator circumference are clearly visible in the figure 19.
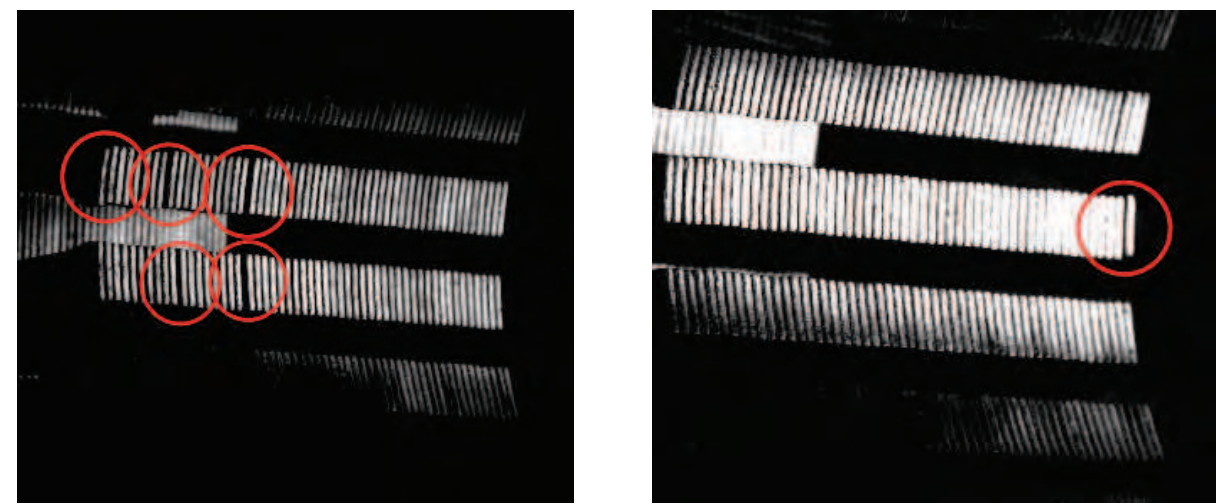

Fig. 19. Slots and plate derivations on the inner stator circumference

Results of the detection of spherity, height/width, parallelism and perpendicularity of the stator package are shown in figure 20. In figure 20a an example of (non)spherity and the height/width of the stator is visible. An example of successful detection of parallelism and perpendicularity of the stator package is shown in figure $20 \mathrm{~b}$. In both cases a satisfactory result is achieved only with a telecentric lens.

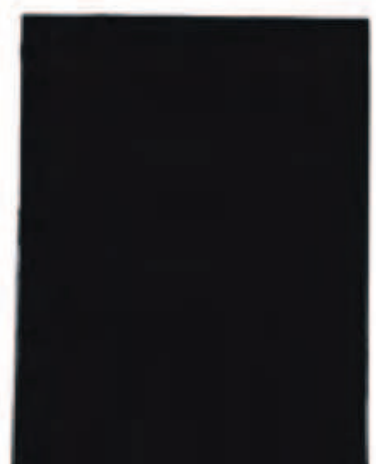

a)

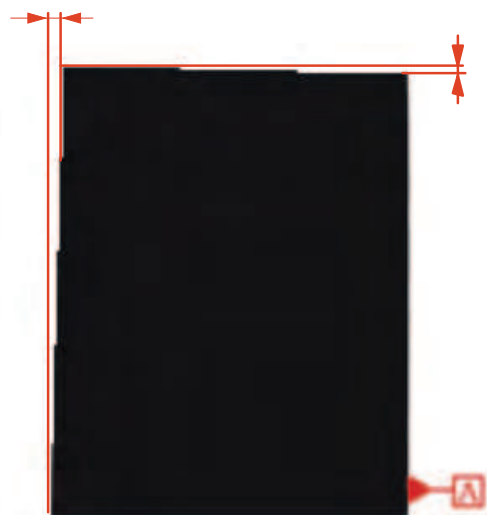

b)

Fig. 20. Successful detection of: a) (non)spherity, the height and b) parallelism and perpendicularity.

The results of the experimental analysis of conditions for a successful replacement of manual (done by workers) quality control of EM stator packages by a robot vision control can enable better efficiency and accuracy of the stator quality control process through the unlimited time 
period, independently of different subjective and objective disturbances. At the same time the machine vision control can help with the humanization of the pretentious labour filed.

\subsection{Algorithm development for a diameter and roundness measurement of a welded ring}

Another assembly application, where an intelligent measuring control system with integrated robot vision for enabling the solving of complex assembly and quality control tasks with high accuracy and speed is needed, is the welding of a metal ring. The welding process must satisfy high quality standards and expectations because the diameter of the welded ring $D_{N}$ must reach the accuracy $\pm 0.05 \mathrm{~mm}$ with the repeatability $\pm 0.01 \mathrm{~mm}$ and at the same time the roundness of the welded ring $O_{V}$ must reach the accuracy $\pm 0.25 \mathrm{~mm}$ with the repeatability $\pm 0.05 \mathrm{~mm}$ (figure 21 ). The ring is shown as black points and the minimum (Dmin) and maximum (Dmax) diameters are shown as two thinner red hatched curves. The difference between the maximum and minimum diameters is an indication of the roundness of the ring $(\mathrm{Ov})$. A high level of accuracy and repeatability for the measurement, demands the introduction of a 100-percent control process. The quality-control procedure for a welded ring must involve a visual observation of the inner surface of the welded ring, checking the inner and the outer diameter as well as the roundness of the welded ring.

The application of robot vision in a quality-control procedure for a welded ring is a very complex and purpose-specific task which demands a great amount of expert knowledge, experiences and innovations. Besides the previously described approaches and conditions for an effective robot vision application, the development of the mathematical algorithm, which enables the use of robot vision for the diameter and the roundness measurement of welded rings is one of the major challenges in this case. Even greater challenge represents the demand, that the measuring system must satisfy all the demands for the successful implementation in industrial application.

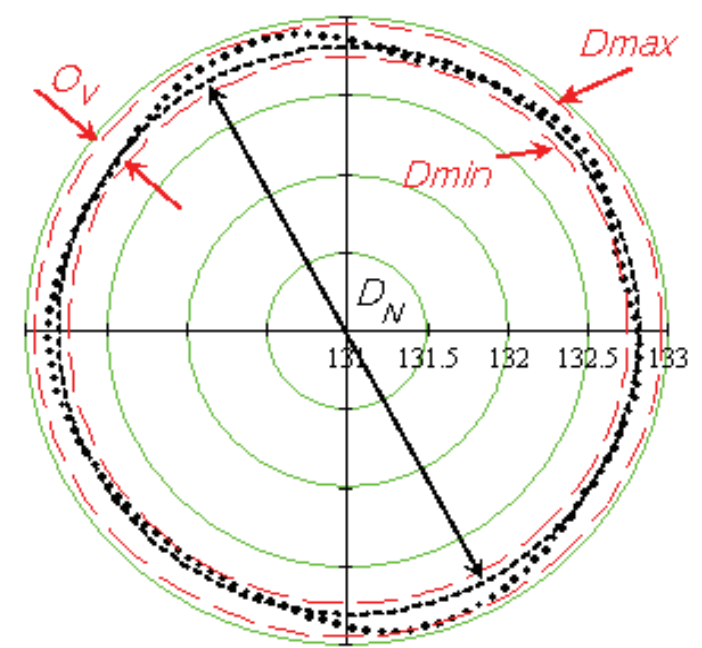

Fig. 21. Graphical representation of the measuring values, ring curve 
In the past few years there have been developments of mathematical algorithms for each measurement procedure in the robot vision control process, which is a multilayer problem for every new application of robot vision. A robot vision system which is used in an industrial environment for tasks such as inspection, measurement, and fault detection, has to be a robust and very reliable system. For that reason, the development of measurement equipment using robot vision has to follow a fixed procedure (Skvarc, 2000; Trdic, 2000). Usually, the procedure is split into a precise determination of tasks (measuring, fault detection) and goals, into the robot vision and working conditions selection (illumination and position determination), component selection of the machine vision (camera, computer, lenses and optics), and, finally, the development of an automatic robot handling system for the parts.

Main idea of the control procedure is the non-contact control method using robot vision, which involves cameras and laser diodes mounted on a solid holder and a rotating table, presented in figure 22 (Petrisic, 2008).

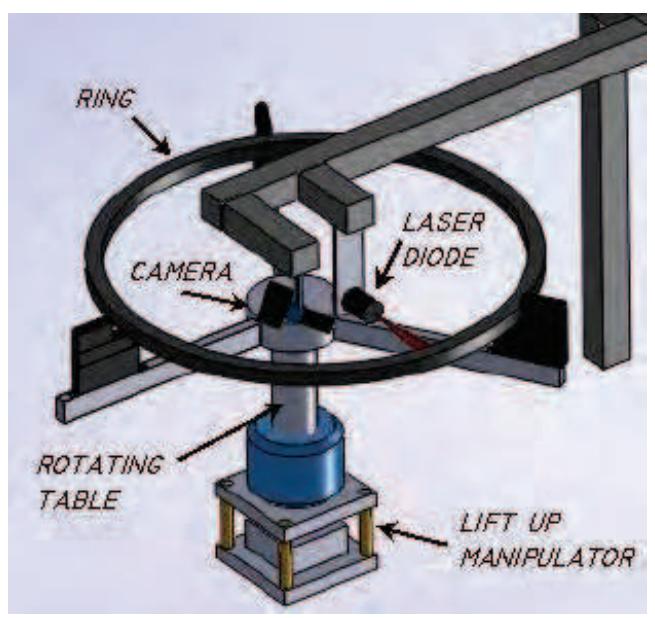

Fig. 22. Measuring system configuration

The ring is placed on the table, which is rotating during the measuring process. The laser path (the vertical lighting line on the inner surface of the ring) is observed with a camera (Pauli, 2001; Redford, 1986). A lift-up manipulator is used when the ring is put on and off the table. The heart of the process is the FDS Imaging Vision software with the integrated numerical program VBA (Visual Basic for Application). After choosing laser triangulation method (Demeyere, 2006) as the measuring process, it is important to analyze a mathematical description of the ring's trajectory and to develop the main algorithm for the eccentric placement elimination. The final step is the development of the proper mathematical algorithm, which is then integrated into the VBA program (Simic et al., 2009).

Measuring the surface of a complex relief by using the laser triangulation method is a wellknown and often-used process. The laser path and the correctly oriented camera are shown in figure 23a. Moving the laser path and the camera together along the surface makes it 
possible to measure the relief. This principle is used also in our case for measuring the shape (the diameter and roundness) of the welded ring (figure 23b).

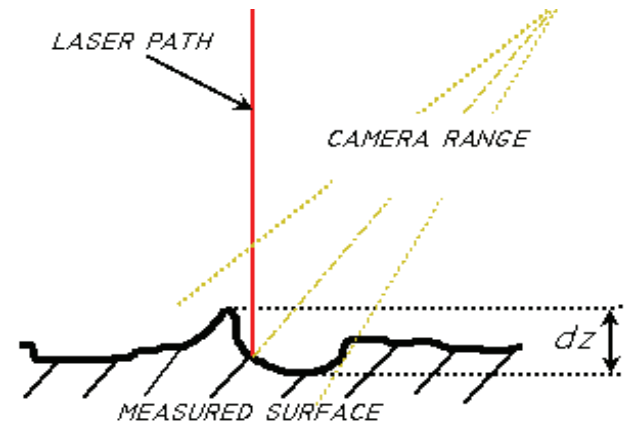

a)

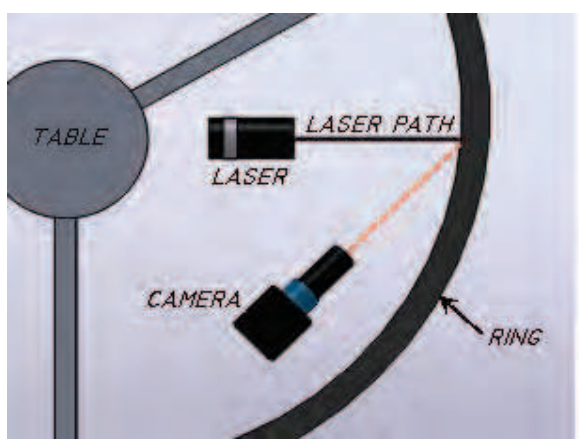

b)

Fig. 23. a) Surface measurement using laser triangulation; b) Laser triangulation method, ring movement

The measuring configuration consists of a single camera and a single laser diode, which are then mounted in the fixed holder. The welded ring is placed - but not fixed - on the rotating table without centering it. The laser path is directed to the inside of the ring's surface, so it is possible to get moving the vertical lighting lines by moving the ring edge along the laser's path. This laser line is observed using a CCD camera, which has to be set up correctly. A 30 to 60 degree angle between the camera (optical axis) and the laser path is needed to achieve the optimum conditions (Hecht, 1987; Gruen, 2001).

When the welded ring is rotating, the observing line or point moves along the laser path, and what we see on the camera screen is shown in figures $24 \mathrm{a}$ and $\mathrm{b}$.

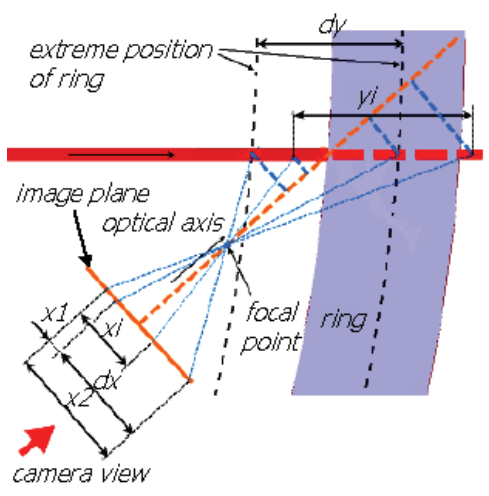

a)

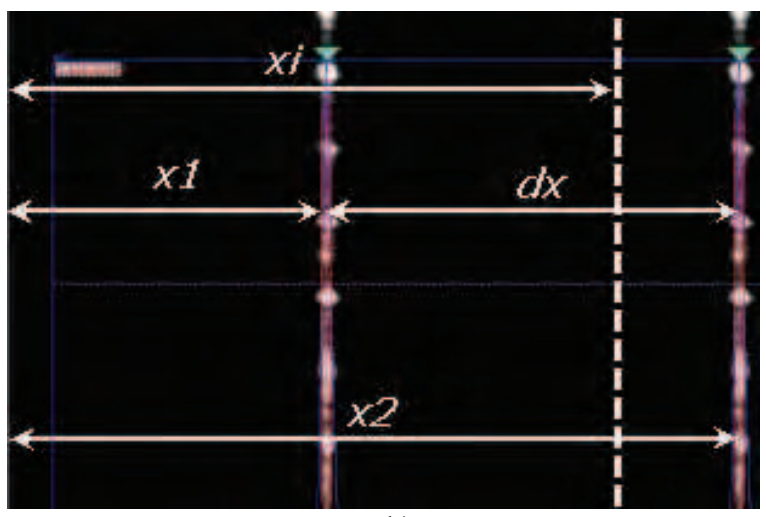

b)

Fig. 24. a) Principle of the laser triangulation method; b) Camera view, calibration on the camera screen, FDS Imaging software interface 
During a single turn of the ring, one hundred photos are taken (the camera grabs one image for every 3.6 degrees of rotation). The whole camera process can be referred to as image processing. The camera image, presented on the main display of the computer, is shown in figure 24b. The laser line, shown as a white vertical line, is moving right and left. For a better presentation and understanding of the problem, two white lines are sketched on the screen (see figure 24b). They represent the extreme right and left positions of the laser line (the extreme right and left positions of the welded ring) while the ring is rotating.

All the parameters that are needed for a triangulation-method calculation are presented in figure 24a. The transformation equation for the presented triangulation method, which defines the deviation of the ring from the previously defined parameters, is given by eq. (1):

$$
y_{i}=\left(x_{i}-x_{1}\right) \cdot k
$$

On the basis of eq. (1) it is possible to calculate the movement of a ring $y_{i}$ by knowing the parameters $x_{i}$ (the actual movement of the laser line), $x_{1}$ (the extreme left position of the laser line, used for the scale calibration) and the transformation factor $k$, known as the scale factor, which can be calculated by using eq. (2) (see figure $24 a$ ).

$$
k=\frac{d y}{d x}
$$

Theoretical and mathematical descriptions of the measuring-point trajectory are treated in detail by Simic (Simic et al., 2009). It is important to emphasize that the measuring system, which is described in figure 22 , needs to be ideal, which means:

- the axis of the rotating table is ideal, i.e., it is without any oscillations during the rotation,

- the welded ring and the rotating table are treated as a rigid body.

When an industrial measuring principle is set as the final goal, it is clear that it is impossible to place the welded ring exactly on the rotating axle. For this reason the movement of the measuring point for the ideal circular ring has to be especially considered, where the centre of the ring is placed outside the rotating axle, which represents the origin of the $(x, y)$ global coordinate system (see figure 25a). When the ring is rotating, the centre of the ring moves along the centre trajectory, as shown in figure $25 \mathrm{~b}$.

For this case the laser line's movement on the ring is described by eq. (3), where $R_{i}(\alpha)$ represents the variable diameter of the ring, depending on the rotating angle $\alpha$. Figure $25 c$ presents the results of the points $R_{i}(\alpha)$, calculated using eq. (3).

$$
R_{i}(\alpha)=E \cdot \cos \alpha_{i}+\sqrt{R_{v}^{2}-\left(E \cdot \sin \alpha_{i}\right)^{2}}
$$

using the parameters: $E \ldots$ the eccentricity of ring $[\mathrm{mm}], R_{V \ldots}$ the radius of ring $[\mathrm{mm}], a$...the angle of rotation $\left[^{\circ}\right]$ 


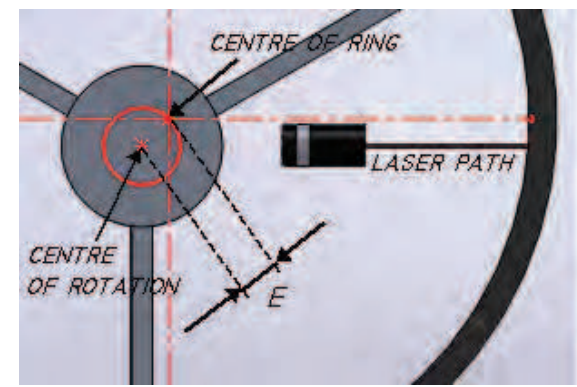

a)

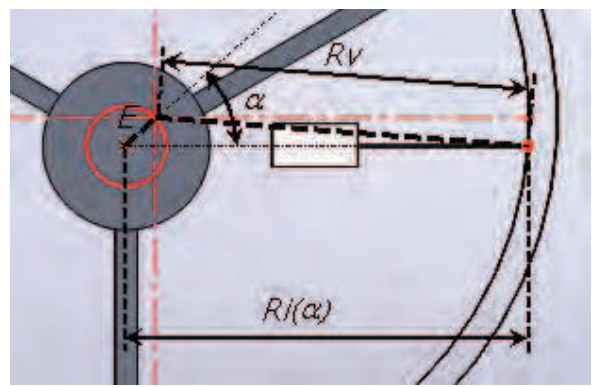

b)

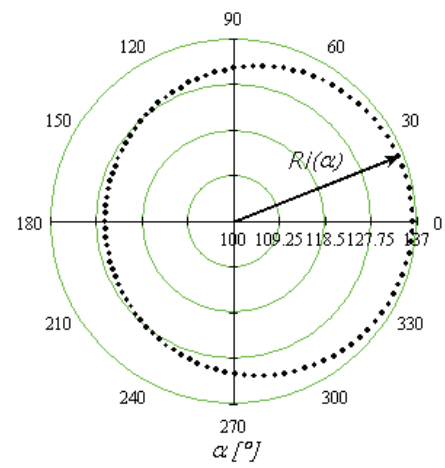

c)

Fig. 25. a) Ideal circular ring centered outside the rotating axle - influence on the value of the diameter; b) Trigonometric representation of the ring projection; $c)$ Sketched ring points $R_{i}(\alpha)$

It is also necessary to consider carefully the influence of the eccentric position of the laser path on the diameter value, as it is shown in figures 26 a and $b$. A critical situation only occurs when the position of the laser is changed during the measuring process or the rotation of the ring. Otherwise, this problem can be eliminated by the software correction of the ring's radius (radius calibration).

If the position of the laser diodes is changed by a distance $\pm z$, the diameter value can be calculated using eq. (4). A comparison between the calculated values of the diameter $R_{i}(\alpha)$ and $R_{z}(\alpha)$ and the dependence on the rotating angle $\alpha$ are shown in figure 26c. It is also necessary to consider the influence of the system vibration during the measuring process (Simic et al., 2009).

$$
R_{z}(\alpha)=E \cdot \cos \alpha_{i}+\sqrt{R_{v}^{2}-\left(E \cdot \sin \alpha_{i}-z\right)^{2}}
$$

where the parameters are as follows:

$E$... the eccentricity of ring $[\mathrm{mm}]$

$R_{V \ldots .}$ the radius of the ring [mm]

$\alpha_{i} \ldots$ the angle of rotation $\left[^{\circ}\right]$

$z$...the eccentricity of the laser path $[\mathrm{mm}]$ 


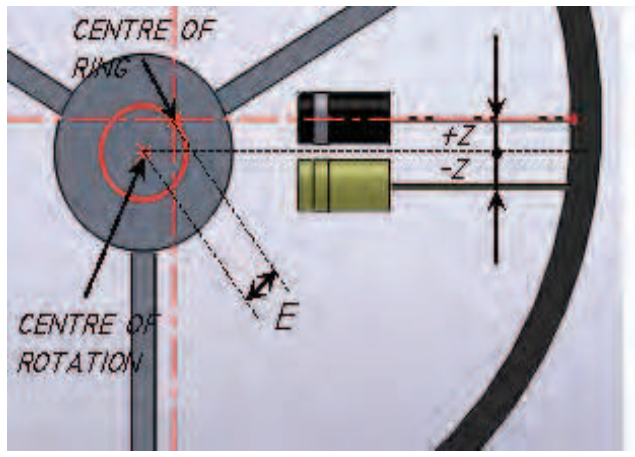

a)

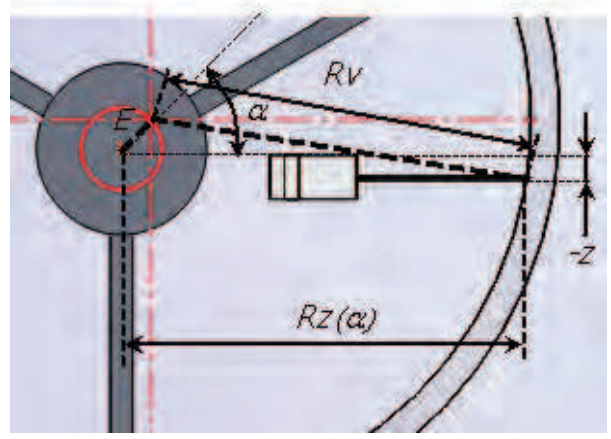

b)

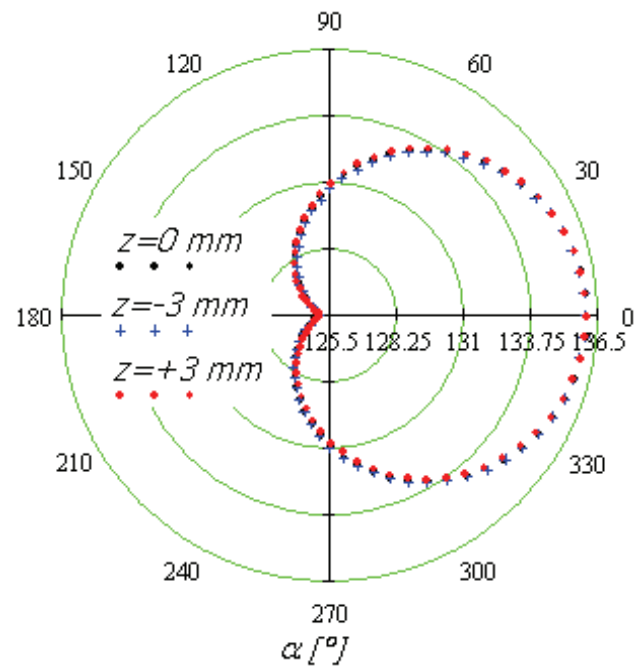

c)

Fig. 26. a) Eccentric position of the laser - influence on the diameter value; b) Graphical analysis of the ring projection; c) Comparison between the $R_{i}(\alpha)$ and the $R_{z}(\alpha)$

One of the possibilities to eliminate the eccentric displacement of the ring is to calculate the area and the centre of this area, which is generated by measuring the points that are subsequently connected by lines to generate a polygon. From knowing the size and the centre point of the area and with further optimization of the mathematical algorithm, it is possible to eliminate the eccentric displacement of the ring on the rotating table (Simic et al., 2009).

By considering the optimized mathematical algorithm, experimental verifications and industrial demands, it is possible to apply the robot vision system for the quality control of the diameter and roundness measurement of the welded ring. The results and graphical explanation of the ring diameter and the ring roundness are presented in figures 27a and $b$. The calculated average radius or diameter is shown as a full curve in figure 27a and the 
measuring points, transformed into the origin are shown as the hatched curve, as shown in the same figure. The black points in figure $27 \mathrm{~b}$ represent the real ring shape, while the average diameter curve represents the $x$ axis of the graph (full black line). The difference between the full and hatched curves $(O)$ is given by eq. (5), and is calculated for every single point of the ring circumference at the given angle $\alpha$ :

$$
O(\alpha)=R_{v i}-R_{-} a v g
$$

The minimum $\left(O_{\min }\right)$ and the maximum $\left(O_{\max }\right)$ differences between these two curves are used to calculate the roundness of the ring, $O_{v}$ (Zhao \& Chen, 2005) - equations 6 and 7.

$$
\begin{gathered}
O_{\text {min }}(\alpha)=\min \left(R_{v i}-R_{-} a v g\right) \\
O_{\text {max }}(\alpha)=\max \left(R_{v i}-R_{-} a v g\right) \\
O v=\left|O_{\text {min }}(\alpha)\right|+\left|O_{\text {max }}(\alpha)\right|
\end{gathered}
$$

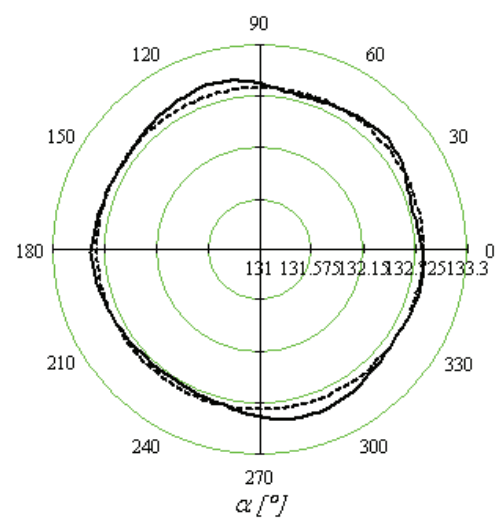

a)

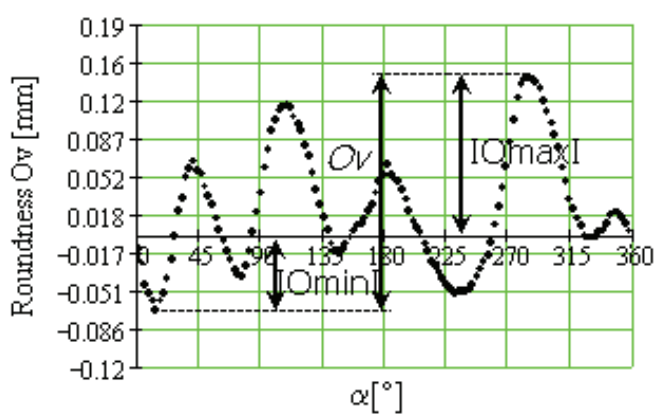

b)

Fig. 27. Results: a) Average and ring diameter curves; b) Roundness of the ring depending on the rotation angle $\alpha\left[^{\circ}\right]$ (Simic et al., 2009)

\section{Conclusion}

The application of robot vision in robot assembly and control processes provides immense potential and challenges, at the same time, for both research and industrial applications, as can be seen by the recent developments summarized in this chapter. It can easily be perceived that the next generation of assembly technology will include versatile robot vision systems with a high level of versatility and robustness. The chapter provides a brief overview of the most recent research efforts in the area of machine vision in assembly and control processes. Various approaches and issues relating assembly, robot assembly, robot vision and object recognition including the bin picking problem are addressed. 
In the first part of the chapter, a general overview and the state-of-the art of the robot vision implementation in the filed of assembly and quality control processes is given, considering robot vision with its advantages and disadvantages and the structure of a typical robot vision system with its basic components description. Some methods, most commonly used in the robot vision, are briefly described and some recognition principles of objects as well as the depth estimation techniques are presented, discussed and some researches are introduced.

In the second part of the chapter, object recognition in assembly and quality control processes is discussed. In this regard all key components of an object recognition system are presented and discussed more in detail by giving an overview of different research works and published papers considering various algorithms for effective object recognition. Also the bin-picking problem with randomly organized 3-D parts in a bin is treated.

In the third or last part of the chapter the implementation of robot vision in control processes is discussed more in detail by considering handling, measurement, fault detection etc. of objects in laboratory and in industrial environment. Additionally, some examples of algorithm development and its practical implementation supported with research results are presented and discussed.

The impact of the illumination on the accuracy of the quality control using the robot vision in the case of the EM stator package assembly process is presented more in detail. Some experimental methods of the identification of conditions and lighting techniques are presented, which decisively influence the practical introduction of the robot vision in the process of quality control in the EM stator assembly. The experimental results prove that the reliable replacement of the manual quality control with the robot vision control in the assembly of the EM stator depends decisively on the right choice of the techniques and the type of illumination of the object. The chapter ends with the presentation and discussion of the results of the research work, focused on the development of a mathematical-numerical algorithm for a modern robot-vision measuring system. A robot-vision experimental system for the ring-diameter and ring-roundness measurements is presented.

\section{References}

Alter, T., Basri, R. (1998). Extracting salient curves from images: An analysis of the saliency network, International Journal of Computer Vision, Volume 27, Issue 1 (March 1998), pp. 51 - 69, ISSN:0920-5691

Antrade-Cetto, J. \& Kak, A.C. (2000). Object Recognition, Wiley Encyclopedia of Electrical Engineering, J.G. Webster ed., John Wiley \& Sons, Sup. 1, 2000, pp. 449-470

Baba, M., Narita, D. \& Ohtani, K. (2004). A New Method of Measuring the 3-D Shape and Surface Reflectance of an Object Using a Laser Rangefinder, Proceedings of Instrumentation and Measurement Technology Conference IMTC 2004, Corno, Italy, 2004

Balslev, I. \& Eriksen R.D. (2002). From belt picking to bin picking, Proceedings of SPIE - The International Society for Optical Engineering, 4902:616-623, 2002 
Batchelor, B.G. \& Whelan, P.F. (2002). Intelligent Vision Systems for Industry, e-book, ivsi@cs.cf.ac.uk, 2002

Batchelor, B.G. (2006). Natural and artificial vision, Cardiff University, http:// bruce.cs.cf.ac.uk/index

Boothroyd, G. (2005). Assembly Automation and Product Design, CRC Press, 2005

Boughorbel, F., Zhang, Y., Kang, S., Chidambaram, U., Abidi, B., Koschan, A. \& Abidi, M. (2003). Laser ranging and video imaging for bin picking, Assembly Automation, Vol. 23, No. 1, 2003, pp. 53-59

Braggins, D. (2006). Vision today for assembly automation, Assembly Automation 2, 6/2 (2006), pp. $181-183$.

Brian, H. (2008). What's Next for Robotics, Robotic Industries Association, www.robotics.org, October 2008

Brumson, B. (2009). Seeing and Moving: A Preview of the 2009 International Robot, Vision and Motion Control Show, www.robotics.org, April 2009

Burns, R. A. (1999). Machine vision lighting Techniques for electronics assembly, RVSI Northeast Robotics, http://www.machinevisiononline.org/public/articles/RVSI_LightingforMachineVision.pdf

Cecil, J., Powell, D. \& Vasquez, D. (2007). Assembly and manipulation of micro devices - A state of the art survey, Robotics and Computer Integrated Manufacturing, 23 (2007), pp. 580-588, www.elsevier.com

Christe, B. (2009). Robotic Application Research: Past, Present, \& Future, www.robotics.org, July 2009

Cox, I., Rehg, J. \& Hingorani, S. (1993). A bayesian multiple-hypothesis approach to edge grouping and contour segmentation, IJCV 1993, 11(1): pp. 5-24

Davies, E.R. (2005). Machine vision: Theory. Algorithms. Practicalities., Elsevier/Morgan Kaufman Publishers, ISBN 0-12-206093-8

Faugeras, O. (1993). Three-Dimensional Computer Vision - A Geometric Viewpoint, The MIT Press, 1993,

Fischler, M. \& Bolles, R. (1986). Perceptual organization and curve partitioning, PAMI 1986, 8(1): pp. 100-105

Forsyth, D.A. \& Ponce, J. (2002). Computer Vision: A Modern Approach, Prentice Hall, 2002

Goldfeder, C., Allen, P.K., Lackner, C. \& Pelossof, R. (2007). Grasp planning via decomposition trees, IEEE ICRA - International Conference on Robotics and Automation, Rome, 2007

Griot, M. (2009) Machine vision, www.mellesgriot.com/products/machinevision/, July 2009

Gruen, A. (2001). Calibration and orientation of cameras in computer vision, Springer, Berlin, 2001

Guichard, F. \& Tarel J.P. (1999). Curve Finder Perceptual Gruping and a Kalman Like Fitting, Proceedings of the 7th IEEE International Conference on Computer Vision, Kerkyra, Greece, September 1999, pp 1003-1008

Gupta, M.M. \& Knopf, G. (1993). Neuro-Vision Systems: A Tutorial, a selected reprint, IEEE Press, Volume IEEE, Neural networks Council Sponsor, New York, 1993

Handelsman, M. (2006). Vision, tactile sensing simplify many tasks, www.industrialcontroldesignline.com

Hecht, E. (1987). Optics, Addison-Wesley, 2001 
Hema, C.R., Paulraj, M.P., Nagarajan, R. \& Sazali Y. (2007). Segmentation and Location Computation of Bin Objects, International Journal of Advanced Robotic Systems, Vol 4., No. 1 (2007), pp. 57-62

Herakovic, N. (2007) a. Computer and Machine Vision in Robot-based Assembly, Journal of Mechanical Engineering, Vol. 53, Nr. 12, 12/2007, pp. 858-871, ISSN 0039-2480

Herakovic, N. (2007) b. An experimental analysis of the conditions for machine vision quality control for FEM stator assembly, Ventil, Vol. 13, Nr. 5, Oct. 2007, pp. 324329, ISSN 1318-7279

Hou. Z.-G., Song, K.-Y., Gupta, M. \& Tan, M. (2007). Neural Units with Higher-Order Synaptic Operations for Robotic Image Processing Applications, Springer, Soft Computing, 2007, Vol. 11, Nr. 3, pp. 221-228

http:// bruce.cs.cf.ac.uk/index

Huang, S.-J. \& Tsai, J.-P. (2005). Robotic automatic assembly system for random operating condition,

International Journal of Advanced Manufacturing Technology, 27 (2005), pp. 334-344, ISBN 0-262-06158-9

Jain, A.K. \& Dora C. (2000). 3D object recognition: Representation, Statistics and Computing, 10 (2000), pp. 167-182

Jenny, R. (2000). Fundamentals of optics-An Introduction for Beginners, Journal of Computer Vision, 40(2) 2000, pp. 142-167

Kellett, P. (2009). Roadmap to the Future, www.robotics.org, August 2009

King, F.G., Puskorius, G.V., Yuan, F., Meier, R.C., Jeyabalan, V. \& Feldkamp, L.A. (1988). Vision guided robots for automated assembly, IEEE, Proceedings of International Conference on Robotics and Automation, Volume 3, 1988, pp. $1611-1616$

Kirkegaard, J. \& Moeslund, T.B. (2006). Bin-Picking based on Harmonic Shape Contexts and Graph-Based Matching, The 18th International Conference on Patern Recognition (ICPR'06), 2006 IEEE

Kirkegaard, J. (2005). Pose Estimation of Randomly Organized Stator Housings using Structured Light and Harmonic Shape Context, Master Thesis, Aalborg University, Denmark, 2005

Klobucar, R., Pacnik, G. \& Safaric, R. (2007). Uncalibrated visual servo control for 2 DOF parallel manipulator

Kristensen, S., Estable, S., Kossow, M. \& Broesel, R. (2001). Bin-picking with a solid state range camera, Robotics and Autonomous Systems, 35 (2001), pp. 143-151

Leumann, M. (2009). Trends in Industrial Image Processing, Vision $\mathcal{E}$ sensors, www.visionsensorsmag.com, March 2009

Lowe, D.G. (1987). Three-Dimensional Object Recognition from Single Two-Dimensional Images, Artificial Intelligence, 31, 3(1987), pp. 355-395

Merva, J.J. (2009). What Every Machine Vision User Should Know About Lighting, www.machinevisiononline.org, July 2009

Motai, Y. \& Kosaka, A. (2004). Concatenate Feature Extraction for Robust 3D Elliptic object Localization, Symposium on Applied Computing, ACM 2004, Cyprus, 2004

Nof, S. Y., Wilhelm, W. E. \& Warnecke, H-J. (1997). Industrial Assembly, Chapman \& Hall, ISBN 0412557703 
Ostojic, G.; Lazarevic, M.; Stankovski, S.; Cosic, I. \& Radosavljevic, Z. (2008). Radio Frequency Identification Technology Application in Disassembly Systems, Journal of Mechanical Engineering, Vol. 54, Nr. 11, pp. 759-767.

Pauli, J. (2001). Learning - Based Robot Vision, Springer, 2001

Peña-Cabrera, M., Lopez-Juarez, I, Rios-Cabrera, R. \& Corona-Castuera J. (2005). Machine vision approach for robotic assembly, Assembly Automation, 25/3 (2005), pp. 204-216

Petrisic, J., Suhadolnik, A. \& Kosel F., (2008). Object length and area calculations on the digital image, Proceedings of 12th International Research/Expert Conference Trends in the Development of Machinery and Associated Technology TMT 2008, Istanbul, Turkey, 2630 August, 2008.

Pope, A. (1994). Learning Object recognition Models from Images, Ph.D. research Proposal, University of British Columbia

Pope, A.R. \& Lowe D.G. (1996). Learning appearance models for object recognition, Proceedings of International Workshop on Object Representation for Computer Vision, Springer, Berlin, 1996, pp. 201-219

Pope, A.R. \& Lowe D.G. (2000). Probabilistic Models of Appearance for 3-D Object Recognition, International Journal of Computer Vision, Volume 40, Number 2, November 2000 , pp. 149-167(19)

Rampersad, H. K. (1994). Integrated and Simultaneous design for Robotic Assembly, John Wiley E Sons, England, ISBN 0471950181

Ray, L.P. (1990). Monocular 3D Vision for a Robot Assembly, International Conference on Systems Engineering, IEEE, 1990

Redford, A. H. (1986). Robots in assembly, Open University Press, 1986, ISBN: 033515400X

Rosin, P. \& West, G. (1995). Nonparametric segmentation of curves into various representations, PAMI 1995, 17(12): pp. 1140-1153

Roth, D., Yang, M.-H. \& Ahuja N. (2002). Learning to recognize Objects, Neural Computation $14 / 2002$

Rowland, J. J. \& Lee, M. H. (1995). Intelligent assembly systems, World Scientific, ISBN 981022494X

Saxena, A, Chung, S.H. \& Ng, A.Y., (2007) a. 3-D Depth reconstruction from a Single Still Image, International Journal of Computer Vision (IJCV), Aug. 2007

Saxena, A, Schulte, J. \& Ng, A.Y., (2007) b. Depth Estimation using Monocular and Stereo Cues, Proceedings of 20th International Joint Conference on Artificial Intelligence (IJCAI), 2007

Scharstein, D. \& Szelinski, R. (2002). A Taxonomy and Evaluation of Dense Two-Frame Stereo Correspondence Algorithms, International Journal of Computer Vision, $47(1 / 2 / 3) 2002$, pp. 7-42

Schraft, R.D., Ledermann, T. (2003). Intelligent picking of chaotically stored objects, Assembly Automation, Vol.23, Nr. 1, 2003, pp. 38-42

Shapiro, L.G. \& Stockman, G.C. (2001). Computer Vision, Prentice Hall, 2001

Sharma, R. \& Srinivasa, N. (1996). A framework for robot control with active vision using neural network based spatial representation, Proceedings of the 1996 IEEE International Conference on Robotics and Automation, Mineapolis, 1996 
Simic, M., Trdic, F., Skvarc, J. \& Herakovic, N. (2009).Diameter and roundness measurement principle of the welded ring in the control process using the robot vision, Proceedings of $18^{\text {th }}$ International Workshop on Robotics in Alpe-Adria-Danube Region RAAD 09, ISSN 2066-4745, Brasov, May 2009

Skvarc, J. (2000). Solving an industrial problems using the machine vision as an universal appliance, FDS Research company, 2000

Solina, F. (2006). Racunalniski vid nekdaj in danes, Proceedings of the conference ROSUS 2006 Maribor, pp. 3-12, Maribor, 2006 (in Slovene language)

Thorsley, M., Okouneva, G. \& Karpynczyk J. (2004). Stereo Vision Algorithm for Robotic Assembly Operations, Proceedings of First Canadian Conference on Computer and Robot Vision (CRV 2004)

Trdic, F. (2000). Vision Technologies and Neuro Inspectors Training Course, FDS Research company, 2000

Trucco, E. \& Verri, A. (1998). Introductory Techniques for 3-D Computer Vision, Prentice Hall, 1998

Vujovic, I., Petrovic I. \& Kezic D. (2007). Wavelet-Based Edge Detection for Robot Vision Applications, Proceedings of 16th International Workshop on Robotics in Alpe-AdriaDanube Region - RAAD 2007, Ljubljana, 2007

West, P. (2006). A Roadmap for building a Machine Vision System, Automated Vision System, http://www.imagenation.com/pdf/roadmap.pdf

West, P.C. (2009). Choosing the Right Vision Technology, Vision \& Sensors, June 2009, http://www.visionsensorsmag.com/Articles/

Winkler, S., Wunsch, P. \& Hirzinger, G. (1997). A Feature Map Approach to Real-Time 3-D Object Pose estimation from Single 2-D Perspective Views, Proceedings of 19. DAGM Symposium, 1997

with neural network, Proceedings of 16th International Workshop on Robotics in Alpe-AdriaDanube Region - RAAD 2007, Ljubljana

Yli-Jaaski, A. \& Ade, F. (1996). Grouping symmetrical structures for object segmentation and description, Computer Vision and Image Understanding, 63(3) 1996, pp. 399-417

Zhao, J. W. \& Chen, G. Q. (2005). Roundness error assessment, Institute of physics Publishing, $13 / 2005$ 


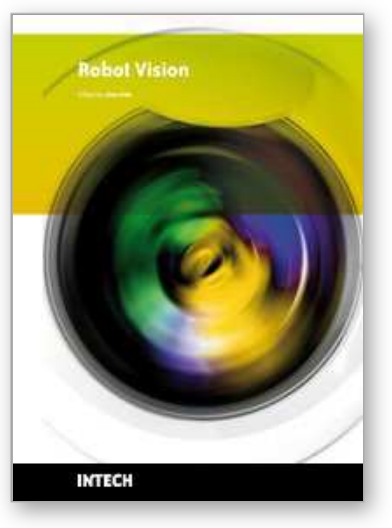

\author{
Robot Vision \\ Edited by Ales Ude
}

ISBN 978-953-307-077-3

Hard cover, 614 pages

Publisher InTech

Published online 01, March, 2010

Published in print edition March, 2010

The purpose of robot vision is to enable robots to perceive the external world in order to perform a large range of tasks such as navigation, visual servoing for object tracking and manipulation, object recognition and categorization, surveillance, and higher-level decision-making. Among different perceptual modalities, vision is arguably the most important one. It is therefore an essential building block of a cognitive robot. This book presents a snapshot of the wide variety of work in robot vision that is currently going on in different parts of the world.

\title{
How to reference
}

In order to correctly reference this scholarly work, feel free to copy and paste the following:

Niko Herakovic (2010). Robot Vision in Industrial Assembly and Quality Control Processes, Robot Vision, Ales Ude (Ed.), ISBN: 978-953-307-077-3, InTech, Available from: http://www.intechopen.com/books/robotvision/robot-vision-in-industrial-assembly-and-quality-control-processes

\section{INTECH}

open science | open minds

\section{InTech Europe}

University Campus STeP Ri

Slavka Krautzeka 83/A

51000 Rijeka, Croatia

Phone: +385 (51) 770447

Fax: +385 (51) 686166

www.intechopen.com

\section{InTech China}

Unit 405, Office Block, Hotel Equatorial Shanghai

No.65, Yan An Road (West), Shanghai, 200040, China

中国上海市延安西路65号上海国际贵都大饭店办公楼405单元

Phone: +86-21-62489820

Fax: $+86-21-62489821$ 
(C) 2010 The Author(s). Licensee IntechOpen. This chapter is distributed under the terms of the Creative Commons Attribution-NonCommercialShareAlike-3.0 License, which permits use, distribution and reproduction for non-commercial purposes, provided the original is properly cited and derivative works building on this content are distributed under the same license. 